\title{
Refined existence and regularity results for a class of semilinear dissipative SPDEs
}

\author{
Carlo Marinelli* $\quad$ Luca Scarpa ${ }^{\dagger}$
}

December 18, 2019

\begin{abstract}
We prove existence and uniqueness of solutions to a class of stochastic semilinear evolution equations with a monotone nonlinear drift term and multiplicative noise, considerably extending corresponding results obtained in previous work of ours. In particular, we assume the initial datum to be only measurable and we allow the diffusion coefficient to be locally Lipschitz-continuous. Moreover, we show, in a quantitative fashion, how the finiteness of the $p$-th moment of solutions depends on the integrability of the initial datum, in the whole range $p \in] 0, \infty[$. Lipschitz continuity of the solution map in $p$-th moment is established, under a Lipschitz continuity assumption on the diffusion coefficient, in the even larger range $p \in[0, \infty[$. A key role is played by an Itô formula for the square of the norm in the variational setting for processes satisfying minimal integrability conditions, which yields pathwise continuity of solutions. Moreover, we show how the regularity of the initial datum and of the diffusion coefficient improves the regularity of the solution and, if applicable, of the invariant measures.
\end{abstract}

AMS Subject Classification: Primary: 60H15, 47H06, 37A25. Secondary: 46N30.

Key words and phrases: stochastic evolution equations, singular drift, variational approach, monotonicity methods, invariant measures.

\section{Introduction}

We consider semilinear stochastic partial differential equations on a smooth bounded domain $D \subseteq \mathbb{R}^{d}$ of the form

$$
d X(t)+A X(t) d t+\beta(X(t)) d t \ni B(t, X(t)) d W(t), \quad X(0)=X_{0},
$$

where $A$ is a coercive maximal monotone operator on (a subspace of) $H:=L^{2}(D), \beta$ is a maximal monotone graph in $\mathbb{R} \times \mathbb{R}$ defined everywhere, $W$ is a cylindrical Wiener process on a separable Hilbert space $U$, and $B$ is a process taking values in the space of Hilbert-Schmidt operators from $U$ to $L^{2}(D)$ satisfying a (local) Lipschitz continuity condition. Precise assumptions on the data of the problem are given in $\$ 2$ below.

Assuming that the initial datum $X_{0}$ has finite second moment and the diffusion coefficient $B$ is globally Lipschitz continuous, we proved in 24 that equation (1.1) admits a unique solution, in a generalized variational sense, whose trajectories are weakly continuous in $H$. The contribution of this work is to extend these results in several directions. In fact, we show that the solution $X$ is pathwise strongly continuous in $H$, rather than just weakly continuous. This is possible thanks to an Itô-type formula, interesting in its own right, for the square of the $H$-norm of processes satisfying minimal integrability conditions, in a variational setting extending the classical one by Pardoux [26. The pathwise strong continuity of solutions to stochastic equations with singular drift is often a difficult problem, and it was left as an open problem both in the case of semilinear equations (see [3]) as well as of porous media equations (see [5]). This trajectorial regularity result is essential to prove that the Markovian semigroup generated by the solution to (1.1) is strongly continuous, for which we refer to [20.

*Department of Mathematics, University College London, Gower Street, London WC1E 6BT, United Kingdom. URL: http://goo.gl/4GKJP

${ }^{\dagger}$ Faculty of Mathematics, University of Vienna, Oskar-Morgenstern-Platz 1, 1090 Vienna, Austria. E-mail: luca.scarpa@univie.ac.at, URL: https://mat.univie.ac.at/ scarpa/ 
Moreover, the pathwise strong continuity allows us to prove that existence and uniqueness of solutions to (1.1) continues to hold under much weaker assumptions on the initial datum and on the diffusion coefficient. In particular, $X_{0}$ needs only be measurable and $B$ can be locally Lipschitz-continuous with linear growth. Denoting by $\Omega$ the underlying probability space, the solution map $X_{0} \mapsto X$ is thus defined on $L^{0}(\Omega ; H)$, with codomain contained in $L^{0}(\Omega ; E)$, where $E$ is a suitable path space. By the results of 24 we also have that the solution map restricted to $L^{2}(\Omega ; H)$ has codomain contained in $L^{2}(\Omega ; E)$. As a further result, we extrapolate these mapping properties to the whole range of exponents $p \in\left[0, \infty\left[\right.\right.$, that is, we show that if $X_{0} \in L^{p}(\Omega ; H)$ then $X \in L^{p}(\Omega ; E)$ for every positive finite $p$, and we provide an explicit upper bound on the $L^{p}(\Omega ; E)$-norm of the solution in terms of the $L^{p}(\Omega ; H)$ norm of the initial datum. If, in addition, $B$ is Lipschitz-continuous, we show that the solution map is Lipschitz-continuous from $L^{p}(\Omega ; H)$ to $L^{p}(\Omega ; E)$ for all $p \in[0, \infty[$. In the particular case $p=0$, this implies that solutions converge uniformly on $[0, T]$ in probability if the corresponding initial data converge in probability.

The existence of higher moments of the solution in suitable path spaces is essential for various applications, for instance in optimal control problems of stochastic evolution systems of reaction-diffusion type with singular terms. Problems of this type arise naturally in phase-transition modelling and tumor-growth dynamics (see, e.g., [9, 10, 28]). Indeed, a classical way to prove first-order conditions for optimality of a certain given cost functional is to study the differentiability of the so-called control-tostate map. This can be obtained provided that a refined continuous dependence result on the controls holds. To this end, due to the high nonlinearity of the equations involved, one is usually forced to prove boundedness of the solutions in function spaces with higher integrability in $\Omega$ (see, e.g., [28]). For these reasons, the possibility of relating the existence of moments (in probability) of the solutions to the integrability of the data is fundamental. However, while higher-moment estimates are well known for equations with well-behaved nonlinearities, in case of singular potentials as in (1.1) no result is currently available in literature. The possibility of including multivalued graphs $\beta$ as in (1.1) is crucial in diffuse-interface and phase-change modelling, and the results proved in this paper hence constitute a first reference in this direction. Let us also mention that the availability of an $L^{p}$-theory is also crucial in the context of numerical approximation of SPDEs, as for example the convergence rate and the choice of the discretization scheme usually depend on the integrability properties of the solutions (see, e.g., in the context of numerical discretization of SPDEs, 17, Introduction], 14, and [1.

Finally, we show how the smoothness of the solution improves (as well as of invariant measures, if they exist) if the initial datum and the diffusion coefficient are smoother, without any further regularity assumption on the (possibly singular) monotone drift term $\beta$. For example, if $A$ (better said, the part of $A$ in $H$ ) is self-adjoint, the solution has paths belonging to the domain of $A$ in $H$ if $X_{0}$ and $B$, roughly speaking, take values in the domain of $A^{1 / 2}$. This implies that $X$ is a strong solution in the classical sense, not just in the variational one. In particular, if (1.1) represents the abstract formulation of an initial-boundary value problem on $D$, our result provides sufficient conditions on the data implying higher regularity in space of the solution. The main novelty is that this extra regularity can be obtained irrespectively of the bahaviour of the nonlinear term $\beta$, meaning that one can obtain higher regularity in space (thus also better compactness properties) without necessarily "smoothing" out the singularities of $\beta$. Such a result is new in the literature, to the best of our knowledge, and is essential in the context of optimal control problems of stochastic systems in phase-field modelling (see [28]). In fact, on the one hand, equations with singular $\beta$ are necessary to model phase-change phenomena, and, on the other hand, the possibility of proving necessary conditions for optimality hinges on the availability of spatial regularity results. For this reason, our contribution is a first step in this direction, apart of providing new results in the regularity theory of singular dissipative stochastic PDEs.

Let us comment now more in detail on the current available literature.

In the classical variational theory of SPDEs, existence and uniqueness of solutions under a local Lipschitz condition on $B$ and measurability of $X_{0}$ were obtained by Pardoux in [26]. Our results do not follow from his, however, as equation (1.1) cannot be cast in the usual variational setting. Stochastic equations where all nonlinear terms are locally Lipschitz-continuous have been considered in the semigroup approach (see, e.g., [19] and references therein), but our existence results are not covered, as $\beta$ can be discontinuous and have arbitrary growth. Moreover, the properties of the solution map between $L^{p}(\Omega ; H)$ and $L^{p}(\Omega ; E)$ do not seem to have been addressed even in the classical variational setting. On the other hand, the continuity of the solution map in the case $p=0$ for ordinary SDEs in $\mathbb{R}^{n}$ with Lipschitz coefficients has been studied, also with very general semimartingale noise (see, e.g., 
8]).

The techniques used to obtain the above results are mostly classical: we truncate the initial datum and the diffusion coefficient in a suitable way, so that existence of solutions can be proved locally (i.e. on a stochastic interval). Uniform estimates on local solutions allow then to extend them to the whole interval $[0, T]$. It is however worth stressing the fact that these arguments are relatively simple only when applied to classes of equations for which existence of strong solutions with continuous trajectories is known (e.g. to equations with a polynomially growing drift). On the other hand, in the case of singular nonlinearities as in (1.1) several non-trivial difficulties appear: for instance, if only weak pathwise continuity is known (as is the case for (1.1), as well as for other equations - cf. the already mentioned 5, 24), it is not clear at all how to define local solutions.

The theory of stochastic evolution equations with monotone drift has considerably developed since the initial contributions by Pardoux, Krylov and Rozovskiu [18, 26. In fact, while in the deterministic case general well-posedness theories for evolution equations with $m$-accretive or maximal monotone operators are available (see, e.g., 4), the picture is much less complete in the stochastic setting, where a general well-posedness theory is currently out of reach. Nonetheless, several results are available for specific classes of equations. For instance, existence of strong solution has been proved in [1] for stochastic equations on a Hilbert space whose drift is the subdifferential of a potential satisfying a sub-homogeneity assumption (an exponentially growing drift is then not allowed). Probabilistically weak solutions, defined in terms of stochastic variational inequalities, have been shown to exist in [6], with no restrictive assumptions on the potential. Let us also mention, parenthetically, that concepts of solution based on stochastic variational inequalities have been proposed also for equations with drift in divergence form and of fast-diffusion type (see [12, 13]).

All of the above results are not applicable to semilinear equations such as (1.1), if neither growth nor coercivity assumptions on $\beta$ are assumed. Well-posedness in the strong sense and ergodicity properties for this class of equations have been obtained in 20, 22, 24, where no growth conditions on the drift are needed. Several analogous results have also been obtained for fully nonlinear equations in divergence form with singular drift, again without any growth assumptions, in [23, 21, 27.

The rest of the text is organized as follows. In $\$ 2$ we state the main assumptions and we recall the well-posedness result for (1.1) obtained in 24. In $\$ 3$ we prove a generalized Itô formula for the square of the norm, as well as the strong pathwise continuity of solutions. In $\$ 4$ we prove existence and uniqueness of strong variational solutions to (1.1) assuming first that $B$ is locally Lipschitz-continuous with linear growth and that $X_{0}$ is square integrable, hence removing the latter assumption in a second step, allowing $X_{0}$ to be merely measurable. While in the former case solutions have finite second moment, in the latter case one needs to work with processes that are just measurable (in $\omega$ ), so that uniqueness has to be proved in a much larger space. This is achieved by a suitable application of the Itô formula of $\$ 3$ and stopping arguments. In $\$ 5$ we show that $X_{0}$ having finite $p$-th moment implies that the solution belongs to a space of processes with finite $p$-moment as well, with explicit control of its norm. The Lipschitz continuity of the solution map is then established in a particular case. Further regularity of the solution and of invariant measures is obtained in the last section, under additional regularity assumptions on $X_{0}$ and $B$.

\section{Acknowledgments}

The authors thank David Cohen for some comments and bibliographycal suggestions. The first-named author gratefully acknowledges the hospitality of the Interdisziplinäres Zentrum für Komplexe Systeme (IZKS) at the University of Bonn, Germany. The second-named author gratefully acknowledges the Austrian Science Fund (FWF) project M 2876.

\section{Assumptions and preliminaries}

\subsection{Notation and terminology}

Given a Banach space $E$, its (topological) dual will be denoted by $E^{\prime}$. Given a further Banach space $F$, the (Banach) space of linear bounded operators from $E$ to $F$ will be denoted by $\mathscr{L}(E, F)$. If $E$ and $F$ are Hilbert spaces, $\mathscr{L}^{2}(E, F)$ stands for the space of Hilbert-Schmidt operators from $E$ to $F$. We recall that Hilbert-Schmidt operators form a two-sided ideal on linear bounded operators. 
A graph $\gamma$ in $E$ is a subset of $E \times E$ and the domain of $\gamma$ is defined as $\mathrm{D}(\gamma):=\{x \in E: \exists y \in E$ : $(x, y) \in \gamma\}$. We shall identify linear unbounded operators between Banach spaces with their graphs, as usual. If $E$ is a Hilbert space, $\gamma$ is monotone if $\left(x_{1}, y_{1}\right),\left(x_{2}, y_{2}\right) \in \gamma$ implies $\left\langle y_{2}-y_{1}, x_{2}-x_{1}\right\rangle_{E} \geq 0$, where $\langle\cdot, \cdot\rangle_{E}$ is the scalar product in $E$. The notion of maximal monotone graph is immediate once graphs are ordered by inclusion.

We shall use the standard notation of stochastic calculus (see, e.g., 25]). In particular, given a càdlàg process $Y$ with values in a separable Banach space $E$, the process $Y^{*}$ is defined as $Y^{*}(t):=$ $\sup _{s \in[0, t]}\|Y(s)\|$. For notational convenience, we shall also denote the time index as a subscript rather than within parentheses. Moreover, a process $Y$ stopped at a stopping time $S$ is denoted by $Y^{S}$, and the stochastic integral of $K$ with respect to a local martingale $M$ is denoted by $K \cdot M$.

\subsection{Assumptions}

Let $D$ be a bounded domain in $\mathbb{R}^{d}$ with smooth boundary, and $V$ a real separable Hilbert space densely, continuously, and compactly embedded in $H:=L^{2}(D)$. The scalar product and the norm of $H$ will be denoted by $\langle\cdot, \cdot\rangle$ and $\|\cdot\|$, respectively. Identifying $H$ with its dual $H^{\prime}$, the triple $\left(V, H, V^{\prime}\right)$ is a so-called Gelfand triple: the duality form between $V$ and $V^{\prime}$ extends the scalar product of $H$, i.e. $\langle v, w\rangle={ }_{V}\langle v, w\rangle_{V^{\prime}}$ for any $v, w \in H$. For this reason, we shall simply denote the duality form of $V$ and $V^{\prime}$ by the same symbol used for the scalar product in $H$.

The following assumptions on the linear operator $A \in \mathscr{L}\left(V, V^{\prime}\right)$ will be tacitly assumed to hold throughout the whole text:

(i) there exists $C>0$ such that $\langle A v, v\rangle \geq C\|v\|_{V}^{2}$ for every $v \in V$;

(ii) the part of $A$ in $H$ can be extended to an $m$-accretive operator $A_{1}$ on $L^{1}(D)$;

(iii) for every $\delta>0$, the resolvent $\left(I+\delta A_{1}\right)^{-1}$ is sub-Markovian, i.e. for every $f \in L^{1}(D)$ such that $0 \leq f \leq 1$ a.e. on $D$, one has $0 \leq(I+\delta A)^{-1} f \leq 1$ a.e. on $D$;

(iv) there exists $m \in \mathbb{N}$ such that $\left(I+\delta A_{1}\right)^{-m} \in \mathscr{L}\left(L^{1}(D), L^{\infty}(D)\right)$.

We shall occasionally refer to hypothesis (i) as coercivity of $A$, and to hypothesis (iv) as ultracontractivity of the resolvent of $A_{1}$.

Let us now state the assumptions on the nonlinear part of the drift: $\beta \subset \mathbb{R} \times \mathbb{R}$ is a maximal monotone graph such that $0 \in \beta(0)$ and $\mathrm{D}(\beta)=\mathbb{R}$. Let $j: \mathbb{R} \rightarrow[0,+\infty)$ be the unique convex lowersemicontinuous function such that $j(0)=0$ and $\beta=\partial j$, where $\partial$ stands for the subdifferential in the sense of convex analysis. We assume that

$$
\limsup _{|r| \rightarrow \infty} \frac{j(r)}{j(-r)}<\infty
$$

Denoting the Moreau-Fenchel conjugate of $j$ by $j^{*}$, the fact that $\mathrm{D}(\beta)=\mathbb{R}$ is equivalent to the superlinearity of $j^{*}$ at infinity, i.e. to

$$
\lim _{|r| \rightarrow \infty} \frac{j^{*}(r)}{|r|}=+\infty
$$

For a comprehensive treatment of maximal monotone operators and their connection with convex analysis we refer to, e.g., [4. Here we limit ourselves to recalling that, for any maximal monotone graph $\gamma$ on a Hilbert space $E$, its resolvent and Yosida approximation of $\gamma$ are defined as $(I+\lambda \gamma)^{-1}$ and

$$
\gamma_{\lambda}:=\frac{1}{\lambda}\left(I-(I+\lambda \gamma)^{-1}\right),
$$

respectively, that both are continuous operators on $E$, and that the former is a contraction, while the latter is Lipschitz-continuous with Lipschitz constant bounded by $1 / \lambda$.

Let $(\Omega, \mathscr{F}, \mathbb{P})$ be a probability space, endowed with a right-continuous and completed filtration $\left(\mathscr{F}_{t}\right)_{t \in[0, T]}$, on which a cylindrical Wiener process $W$ on a real separable Hilbert space $U$ is defined. The diffusion coefficient

$$
B: \Omega \times[0, T] \times H \rightarrow \mathscr{L}^{2}(U, H)
$$


is assumed to be such that $B(\cdot, \cdot, x)$ is progressively measurable for every $x \in H$, and to grow at most linearly in its third argument, uniformly with respect to the others. That is, we assume that there exists a constant $N$ such that

$$
\|B(t, \omega, x)\|_{\mathscr{L}^{2}(U, H)} \leq N(1+\|x\|)
$$

for all $(\omega, t, x) \in \Omega \times[0, T] \times H$. In addition to this, we shall consider two different assumptions, namely

(B1) $B$ is Lipschitz continuous in its third argument, uniformly with respect to the others, i.e.

$$
\|B(\omega, t, x)-B(\omega, t, y)\|_{\mathscr{L}^{2}(U, H)} \leq N\|x-y\|
$$

for all $(\omega, t) \in \Omega \times[0, T]$ and $x, y \in H$.

(B2) $B$ is locally Lipschitz continuous in its third argument, uniformly with respect to the others, i.e. there exists a function $R \mapsto N_{R}: \mathbb{R}_{+} \rightarrow \mathbb{R}_{+}$such that

$$
\|B(\omega, t, x)-B(\omega, t, y)\|_{\mathscr{L}^{2}(U, H)} \leq N_{R}\|x-y\|
$$

for all $(\omega, t) \in \Omega \times[0, T]$ and $x, y \in H$ with $\|x\|,\|y\| \leq R$.

Finally, $X_{0}$ is assumed to be an $H$-valued $\mathscr{F}_{0}$-measurable random variable.

Let us now define the concept of solution to equation (1.1).

Definition 2.1. A strong solution to (1.1) is a pair $(X, \xi)$, where $X$ is a $V$-valued adapted process and $\xi$ is an $L^{1}(D)$-valued predictable process, such that, $\mathbb{P}$-almost surely,

$$
\begin{gathered}
X \in L^{\infty}(0, T ; H) \cap L^{2}(0, T ; V), \quad \xi \in L^{1}\left(0, T ; L^{1}(D)\right), \\
\xi \in \beta(X) \quad \text { a.e. in }(0, T) \times D,
\end{gathered}
$$

and

$$
X(t)+\int_{0}^{t} A X(s) d s+\int_{0}^{t} \xi(s) d s=X_{0}+\int_{0}^{t} B(s, X(s)) d W(s)
$$

in $V^{\prime} \cap L^{1}(D)$ for all $t \in[0, T]$.

It is convenient to introduce the family of sets $\left(\mathscr{J}_{p}\right)_{p \geq 0}$ as follows:

$$
\mathscr{J}_{p} \subset\left(L^{p}(\Omega ; C([0, T] ; H)) \cap L^{p}\left(\Omega ; L^{2}(0, T ; V)\right)\right) \times L^{p / 2}\left(\Omega ; L^{1}((0, T) \times D)\right.
$$

formed by processes $(\phi, \psi)$ such that $\phi$ is adapted with values in $V, \psi$ is predictable with values in $L^{1}(D), \psi \in \beta(\phi)$ a.e. in $\Omega \times(0, T) \times D$, and $j(\phi)+j^{*}(\psi) \in L^{p / 2}\left(\Omega ; L^{1}((0, T) \times D)\right.$.

The following well-posedness result has been proved in [24. Just for the purposes of this statement, we shall denote the space $\mathscr{J}_{2}$ with $L^{\infty}(0, T ; H)$ in place of $C([0, T] ; H)$ by $\tilde{\mathscr{J}}_{2}$.

Theorem 2.2. If $X_{0} \in L^{2}\left(\Omega, \mathscr{F}_{0} ; H\right)$ and $B$ satisfies the global Lipschitz condition (B1), then there exists a unique strong solution $(X, \xi)$ to (1.1) belonging to $\tilde{\mathscr{J}}_{2}$. Furthermore, the trajectories of $X$ are weakly continuous in $H$ and the solution map

$$
\begin{aligned}
L^{2}(\Omega ; H) & \longrightarrow L^{2}\left(\Omega ; L^{\infty}(0, T ; H)\right) \cap L^{2}\left(\Omega ; L^{2}(0, T ; V)\right) \\
X_{0} & \longmapsto X
\end{aligned}
$$

is Lipschitz-continuous.

Our main result is the following far-reaching extension of Theorem 2.2 under the more general local Lipschitz continuity assumption (B2), for any $X_{0} \in L^{p}\left(\Omega, \mathscr{F}_{0}, \mathbb{P} ; H\right), p \in[0, \infty[$, there exists a strong solution $(X, \xi)$ belonging to $\mathscr{J}_{p}$, which is unique in $\mathscr{J}_{0}$. In particular, the trajectories of $X$ are strongly continuous in $H$. Precise statements and proofs are given in 4 


\section{Pathwise continuity via a generalized Itô formula}

In this section we prove that, under the assumptions of Theorem 2.2 the unique strong solution $(X, \xi)$ in $\mathscr{J}_{2}$ to (1.1) is such that $X$ admits a modification with strongly continuous trajectories in $H$, rather than just weakly continuous. To this purpose, we need a generalized Itô's formula for the square of the norm under minimal integrability assumptions, that will play a fundamental role throughout.

We first need some preparations. Let us recall that the part of $A$ in $H$ is the linear (unbounded) operator on $H$ defined by $A_{2}:=A \cap(V \times H)$. In particular,

$$
\mathrm{D}\left(A_{2}\right)=\{u \in V: A u \in H\} \quad \text { and } \quad A_{2} u=A u \quad \forall u \in \mathrm{D}\left(A_{2}\right) .
$$

It is well known (see, e.g., [2] that $A_{2}$ is closed and that $\mathrm{D}\left(A_{2}\right)$ is a Banach space with respect to the graph norm

$$
\|u\|_{\mathrm{D}\left(A_{2}\right)}^{2}:=\|u\|^{2}+\|A u\|^{2} .
$$

Moreover, $\mathrm{D}\left(A_{2}\right)$ is continuously and densely embedded in $V$.

Lemma 3.1. Let $v \in V$ and $v_{\lambda}:=\left(I+\lambda A_{1}\right)^{-1} v$. Then $v_{\lambda} \rightarrow v$ in $V$ as $\lambda \rightarrow 0$.

Proof. Let $v \in V$ and $\varepsilon>0$ : since $\mathrm{D}\left(A_{2}\right)$ is densely embedded in $V$, we can choose $u \in \mathrm{D}\left(A_{2}\right)$ such that $\|v-u\|_{V}<\varepsilon$. Setting $u_{\lambda}:=\left(I+\lambda A_{1}\right)^{-1} u$, we have

$$
\left\|v-v_{\lambda}\right\|_{V} \leq\|v-u\|_{V}+\left\|u-u_{\lambda}\right\|_{V}+\left\|u_{\lambda}-v_{\lambda}\right\|_{V}
$$

Since $u, v \in V$, we have $u_{\lambda}-v_{\lambda}=\left(I+\lambda A_{2}\right)^{-1}(u-v)$, and recalling that $A_{2}$ is the part of $A$ in $H$ we have

$$
\left(u_{\lambda}-v_{\lambda}\right)+\lambda A\left(u_{\lambda}-v_{\lambda}\right)=u-v,
$$

where the identity holds in $V$ as well. Taking the duality product with $A\left(u_{\lambda}-v_{\lambda}\right) \in V^{\prime}$, by coercivity and boundedness of $A$ it follows that

$$
{ }_{V^{\prime}}\left\langle A\left(u_{\lambda}-v_{\lambda}\right), u_{\lambda}-v_{\lambda}\right\rangle_{V}+\lambda_{V^{\prime}}\left\langle A\left(u_{\lambda}-v_{\lambda}\right), A\left(u_{\lambda}-v_{\lambda}\right)\right\rangle_{V} \geq C\left\|u_{\lambda}-v_{\lambda}\right\|_{V}^{2}+\lambda\left\|A\left(u_{\lambda}-v_{\lambda}\right)\right\|^{2}
$$

and

$$
V^{\prime}\left\langle A\left(u_{\lambda}-v_{\lambda}\right), u\right\rangle_{V} \leq\|A\|_{\mathscr{L}\left(V, V^{\prime}\right)}\left\|u_{\lambda}-v_{\lambda}\right\|_{V}\|u\|_{V}
$$

hence

$$
C\left\|u_{\lambda}-v_{\lambda}\right\|_{V}^{2}+\lambda\left\|A\left(u_{\lambda}-v_{\lambda}\right)\right\|^{2} \leq\|A\|_{\mathscr{L}\left(V, V^{\prime}\right)}\left\|u_{\lambda}-v_{\lambda}\right\|_{V}\|u\|_{V},
$$

which implies that there exists a constant $N>0$, independent of $\lambda$, such that

$$
\left\|u_{\lambda}-v_{\lambda}\right\|_{V} \leq N\|u-v\|_{V}
$$

or, equivalently, that $\left(I+\lambda A_{1}\right)^{-1}$ is uniformly bounded in $V$ with respect to $\lambda$. This implies that

$$
\left\|u_{\lambda}-v_{\lambda}\right\|_{V} \leq N\|u-v\|_{V} \leq N \varepsilon
$$

It remains to estimate the term $\left\|u-u_{\lambda}\right\|_{V}$. Since $u \in \mathrm{D}\left(A_{2}\right)$ and

$$
u_{\lambda}:=\left(I+\lambda A_{1}\right)^{-1} u=\left(I+\lambda A_{2}\right)^{-1} u
$$

one has $u_{\lambda} \in \mathrm{D}\left(A_{2}^{2}\right)$, hence, recalling that $A_{2}$ is the part of $A$ in $H$,

$$
A u_{\lambda}+\lambda A\left(A u_{\lambda}\right)=A u
$$

in $H \hookrightarrow V^{\prime}$. Taking the duality pairing with $A u_{\lambda} \in \mathrm{D}\left(A_{2}\right) \hookrightarrow V$, one has

$$
{ }_{V^{\prime}}\left\langle A u_{\lambda}, A u_{\lambda}\right\rangle_{V}+\lambda_{V^{\prime}}\left\langle A\left(A u_{\lambda}\right), A u_{\lambda}\right\rangle_{V}={ }_{V^{\prime}}\left\langle A u, A u_{\lambda}\right\rangle_{V},
$$

where

$$
\begin{gathered}
{ }_{V^{\prime}}\left\langle A u_{\lambda}, A u_{\lambda}\right\rangle_{V}=\left\|A u_{\lambda}\right\|^{2}, \quad{ }_{V^{\prime}}\left\langle A\left(A u_{\lambda}\right), A u_{\lambda}\right\rangle_{V} \geq C\left\|A u_{\lambda}\right\|_{V}^{2}, \\
{ }_{V^{\prime}}\left\langle A u, A u_{\lambda}\right\rangle_{V}=\left\langle A u, A u_{\lambda}\right\rangle \leq \frac{1}{2}\|A u\|^{2}+\frac{1}{2}\left\|A u_{\lambda}\right\|^{2},
\end{gathered}
$$


hence

$$
\left\|A u_{\lambda}\right\|^{2}+\lambda C\left\|A u_{\lambda}\right\|_{V}^{2} \leq \frac{1}{2}\|A u\|^{2}+\frac{1}{2}\left\|A u_{\lambda}\right\|^{2}
$$

which implies that $\sqrt{\lambda}\left\|A u_{\lambda}\right\|_{V} \leq N\|A u\|$, with a constant $N$ independent of $\lambda$. Therefore, since $u \in \mathrm{D}\left(A_{2}\right)$,

$$
\left\|u_{\lambda}-u\right\|_{V}=\lambda\left\|A u_{\lambda}\right\|_{V} \leq N \sqrt{\lambda}\|A u\|
$$

Choosing $\lambda$ such that $N \sqrt{\lambda}\|A u\|<\varepsilon$, one has then

$$
\left\|v_{\lambda}-v\right\|_{V}<(2+N) \varepsilon
$$

from which the conclusion follows by arbitrariness of $\epsilon$.

We recall that (see, e.g., [16]) if two Banach spaces $F$ and $G$ are continuously embedded in a separated topological vector space $E$, their sum $F+G$ is defined as the subspace of $E$

$$
F+G:=\{u \in E: \exists f \in F, g \in G: u=f+g\} .
$$

Endowed with the norm

$$
\|u\|_{F+G}:=\inf _{u=f+g}\left(\|f\|_{F}+\|g\|_{G}\right),
$$

$F+G$ is a Banach space. Similarly, the intersection $F \cap G$ is also a Banach space if endowed with the norm

$$
\|u\|_{F \cap G}:=\|u\|_{F}+\|u\|_{G} .
$$

Moreover, if $F \cap G$ is dense in both $F$ and $G$, then $F^{\prime}$ and $G^{\prime}$ are continuously embedded in $(F \cap G)^{\prime}$, and $(F+G)^{\prime}=F^{\prime} \cap G^{\prime}$. In the following we shall deal with $F:=L^{1}(0, T ; H)$ and $G:=L^{2}\left(0, T ; V^{\prime}\right)$, so that as ambient space $E$ one can simply take $L^{1}\left(0, T ; V^{\prime}\right)$. In this case $F \cap G$ is dense in both $F$ and $G$, hence, by reflexivity of $V$,

$$
\left(L^{1}(0, T ; H)+L^{2}\left(0, T ; V^{\prime}\right)\right)^{\prime}=L^{\infty}(0, T ; H) \cap L^{2}(0, T ; V) .
$$

Theorem 3.2. Let $Y, v$ and $g$ be adapted processes such that

$$
\begin{gathered}
Y \in L^{0}\left(\Omega ; L^{\infty}(0, T ; H) \cap L^{2}(0, T ; V)\right), \\
v \in L^{0}\left(\Omega ; L^{1}(0, T ; H)+L^{2}\left(0, T ; V^{\prime}\right)\right), \\
g \in L^{0}\left(\Omega ; L^{1}\left(0, T ; L^{1}(D)\right)\right), \\
\exists \alpha>0: \quad j(\alpha Y)+j^{*}(\alpha g) \in L^{0}\left(\Omega ; L^{1}((0, T) \times D)\right) .
\end{gathered}
$$

Moreover, let $Y_{0} \in L^{0}\left(\Omega, \mathscr{F}_{0} ; H\right)$ and $G$ be a progressive $\mathscr{L}^{2}(U, H)$-valued process such that

$$
G \in L^{0}\left(\Omega ; L^{2}\left(0, T ; \mathscr{L}^{2}(U, H)\right)\right) .
$$

If

$$
Y(t)+\int_{0}^{t} v(s) d s+\int_{0}^{t} g(s) d s=Y_{0}+\int_{0}^{t} G(s) d W(s) \quad \forall t \in[0, T] \quad \mathbb{P} \text {-a.s. }
$$

in $V^{\prime} \cap L^{1}(D)$, then

$$
\begin{aligned}
\frac{1}{2}\|Y(t)\|^{2} & +\int_{0}^{t}\langle v(s), Y(s)\rangle d s+\int_{0}^{t} \int_{D} g(s, x) Y(s, x) d x d s \\
& =\frac{1}{2}\left\|Y_{0}\right\|^{2}+\frac{1}{2} \int_{0}^{t}\|G(s)\|_{\mathscr{L}^{2}(U, H)}^{2} d s+\int_{0}^{t} Y(s) G(s) d W(s)
\end{aligned}
$$

for all $t \in[0, T]$ with probability one. 
Proof. Since the resolvent of $A_{1}$ is ultracontractive by assumption, there exists $m \in \mathbb{N}$ such that

$$
\left(I+\delta A_{1}\right)^{-m}: L^{1}(D) \rightarrow H \quad \forall \delta>0 .
$$

Using a superscript $\delta$ to denote the action of $\left(I+\delta A_{1}\right)^{-m}$, we have

$$
Y^{\delta}(t)+\int_{0}^{t} v^{\delta}(s) d s+\int_{0}^{t} g^{\delta}(s) d s=Y_{0}^{\delta}+\int_{0}^{t} G^{\delta}(s) d W(s)
$$

where $g^{\delta} \in L^{1}(0, T ; H)$, hence the classical Itô's formula yields, for every $\delta>0$,

$$
\begin{aligned}
\frac{1}{2}\left\|Y^{\delta}(t)\right\|^{2}+\int_{0}^{t}\left\langle v^{\delta}(s), Y^{\delta}(s)\right\rangle d s+\int_{0}^{t} \int_{D} g^{\delta}(s, x) Y^{\delta}(s, x) d x d s \\
=\frac{1}{2}\left\|Y_{0}^{\delta}\right\|^{2}+\frac{1}{2} \int_{0}^{t}\left\|G^{\delta}(s)\right\|_{\mathscr{L}^{2}(U, H)}^{2} d s+\int_{0}^{t} Y^{\delta}(s) G^{\delta}(s) d W(s) .
\end{aligned}
$$

Let us pass to the limit as $\delta \rightarrow 0$. Since the resolvent of $A_{1}$ coincides on $H$ with the resolvent of $A_{2}$, which converges to the identity in $\mathscr{L}(H)$ in the strong operator topology, we immediately infer that

$$
\begin{aligned}
& Y^{\delta}(t) \longrightarrow Y(t) \quad \text { in } H \quad \forall t \in[0, T], \\
& g^{\delta} \longrightarrow g \quad \text { in } L^{1}\left(0, T ; L^{1}(D)\right) \text {, } \\
& Y_{0}^{\delta} \longrightarrow Y_{0} \quad \text { in } H \text {, } \\
& G^{\delta} \longrightarrow G \quad \text { in } L^{2}\left(0, T ; \mathscr{L}^{2}(U, H)\right)
\end{aligned}
$$

where the last statement, which follows by well-known continuity properties of Hilbert-Schmidt operators, also implies

$$
\int_{0}^{t}\left\|G^{\delta}(s)\right\|_{\mathscr{L}^{2}(U, H)}^{2} d s \longrightarrow \int_{0}^{t}\|G(s)\|_{\mathscr{L}^{2}(U, H)}^{2} d s .
$$

Moreover, by the previous lemma we have

$$
Y^{\delta} \longrightarrow Y \quad \text { in } L^{2}(0, T ; V),
$$

and $Y \in L^{\infty}(0, T ; H)$ and the contractivity in $H$ of the resolvent of $A_{1}$ immediately imply, by the dominated convergence theorem, that $Y^{\delta} \rightarrow Y$ weakly* in $L^{\infty}(0, T ; H)$. Therefore, by reflexivity of $V$,

$$
Y^{\delta} \longrightarrow Y \quad \text { weakly* in } L^{\infty}(0, T ; H) \cap L^{2}(0, T ; V) .
$$

Since $v \in L^{1}(0, T ; H)+L^{2}\left(0, T ; V^{\prime}\right)$, we have that $v=v_{1}+v_{2}$, with $v_{1} \in L^{1}(0, T ; H)$ and $v_{2} \in$ $L^{2}\left(0, T ; V^{\prime}\right)$. In this case $v^{\delta}$ has to be interpreted as

$$
v^{\delta}:=\left(I+\delta A_{1}\right)^{-m} v_{1}+(I+\delta A)^{-m} v_{2} .
$$

Note that this is very natural since $A_{1}$ and $A$ coincide on $\mathrm{D}\left(A_{1}\right) \cap V$. By the properties of the resolvent it easily follows that

$$
v_{1}^{\delta} \longrightarrow v_{1} \quad \text { in } L^{1}(0, T ; H) .
$$

Moreover, since $A^{-1} v_{2} \in L^{2}(0, T ; V)$ and $A^{-1} v_{2}^{\delta}=\left(I+\delta A_{2}\right)^{-m} A^{-1} v_{2}$, by Lemma 3.1 we have that $A^{-1} v_{2}^{\delta} \rightarrow A^{-1} v_{2}$ in $L^{2}(0, T ; V)$, hence also, by continuity of $A$,

$$
v_{2}^{\delta} \longrightarrow v_{2} \quad \text { in } L^{2}\left(0, T ; V^{\prime}\right) .
$$

The convergences of $v^{\delta}$ and $Y^{\delta}$ just proved thus imply

$$
\int_{0}^{t}\left\langle v^{\delta}(s), Y^{\delta}(s)\right\rangle d s \longrightarrow \int_{0}^{t}\langle v(s), Y(s)\rangle d s
$$

for all $t \in[0, T]$. 
We are now going to prove that $\left(\left(Y^{\delta} G^{\delta}\right) \cdot W-(Y G) \cdot W\right)_{T}^{*} \rightarrow 0$ in probability. Setting $M_{\delta}:=$ $\left(Y^{\delta} G^{\delta}\right) \cdot W$ and $M:=(Y G) \cdot W$, it is well known that it suffices to show that the quadratic variation of $M_{\delta}-M$ converges to 0 in probability. One has

$$
\begin{aligned}
{\left[M_{\delta}-M, M_{\delta}-M\right] } & =\left\|Y^{\delta} G^{\delta}-Y G\right\|_{L^{2}\left(0, T ; \mathscr{L}^{2}(U, \mathbb{R})\right)}^{2} \\
& \leq\left\|Y^{\delta} G^{\delta}-Y^{\delta} G\right\|_{L^{2}\left(0, T ; \mathscr{L}^{2}(U, \mathbb{R})\right)}^{2}+\left\|Y^{\delta} G-Y G\right\|_{L^{2}\left(0, T ; \mathscr{L}^{2}(U, \mathbb{R})\right)}^{2} \\
& \leq\|Y\|_{L^{\infty}(0, T ; H)}^{2}\left\|G^{\delta}-G\right\|_{L^{2}\left(0, T ; \mathscr{L}^{2}(U, H)\right)}^{2}+\left\|Y^{\delta} G-Y G\right\|_{L^{2}\left(0, T ; \mathscr{L}^{2}(U, \mathbb{R})\right)}^{2}
\end{aligned}
$$

where the convergence to zero of the first term in the last expression has already been proved, and

$$
\left\|Y^{\delta} G-Y G\right\|_{L^{2}\left(0, T ; \mathscr{L}^{2}(U, \mathbb{R})\right)}^{2} \leq \int_{0}^{T}\left\|Y^{\delta}(s)-Y(s)\right\|^{2}\|G(s)\|_{\mathscr{L}^{2}(U, H)}^{2} d s \longrightarrow 0,
$$

by the dominated convergence theorem, because $Y^{\delta} \rightarrow Y$ pointwise in $H$ and $\left\|Y^{\delta}-Y\right\| \leq 2\|Y\| \in$ $L^{\infty}(0, T)$. We have thus shown that

$$
\int_{0}^{\cdot} Y^{\delta}(s) G^{\delta}(s) d W(s) \longrightarrow \int_{0}^{\cdot} Y(s) G(s) d W(s)
$$

in probability, hence $\mathbb{P}$-a.s. along a subsequence of $\delta$.

Finally, it is clear that $Y^{\delta} g^{\delta} \rightarrow Y g$ in measure in $(0, T) \times D$, and that, thanks to the assumptions on $j$,

$$
\pm \alpha^{2} Y^{\delta} g^{\delta} \leq j\left( \pm \alpha Y^{\delta}\right)+j^{*}\left(\alpha g^{\delta}\right) \lesssim 1+j\left(\alpha Y^{\delta}\right)+j^{*}\left(\alpha g^{\delta}\right)
$$

where the second inequality follows from the fact that, thanks to the assumption on the growth of $j$ at $\infty$, there exists a constant $M>0$ such that

$$
j(r) \leq M(1+j(-r)) \quad \forall r \in \mathbb{R} .
$$

Jensen's inequality for sub-Markovian operators (see, e.g., [15]) thus yields

$$
j\left(\alpha Y^{\delta}\right)+j^{*}\left(\alpha g^{\delta}\right) \leq\left(I+\delta A_{1}\right)^{-m}\left(j(\alpha Y)+j^{*}(\alpha g)\right),
$$

so that

$$
\alpha^{2}\left|Y^{\delta} g^{\delta}\right| \lesssim 1+\left(I+\delta A_{1}\right)^{-m}\left(j(\alpha Y)+j^{*}(\alpha g)\right)
$$

Since $j(\alpha Y)+j^{*}(\alpha g) \in L^{1}((0, T) \times D)$ by assumption, the contractivity of the resolvent in $L^{1}(D)$ and the dominated convergence theorem imply that the right-hand side in the last inequality is convergent in $L^{1}((0, T) \times D)$. Hence $\left(Y^{\delta} g^{\delta}\right)_{\delta}$ is uniformly integrable and, by Vitali's theorem,

$$
\int_{0}^{t} \int_{D} g^{\delta}(s, x) Y^{\delta}(s, x) d x d s \longrightarrow \int_{0}^{t} \int_{D} g(s, x) Y(s, x) d x d s
$$

for all $t \in[0, T]$. The proof is thus completed.

As a first important consequence of the generalized Itô formula we show that (the first component of) strong solutions are pathwise strongly continuous in $H$.

Theorem 3.3. Let $(X, \xi)$ be the unique strong solution to (1.1) belonging to $\mathscr{J}_{2}$. Then $X$ has strongly continuous paths in $H$, i.e. there exists $\Omega^{\prime} \in \mathscr{F}$ with $\mathbb{P}\left(\Omega^{\prime}\right)=1$ such that

$$
X(\omega) \in C([0, T] ; H) \quad \forall \omega \in \Omega^{\prime} .
$$

Proof. Let $r \in[0, T]$. We have to prove that $X(t) \rightarrow X(r)$ in $H$ as $t \rightarrow r, t \in[0, T]$. It follows from Theorem 3.2 that for every $t \in[0, T]$ there exists $\Omega^{\prime} \in \mathscr{F}_{0}$ with $\mathbb{P}\left(\Omega^{\prime}\right)=1$ such that

$$
\begin{aligned}
\frac{1}{2}\|X(t)\|^{2}-\frac{1}{2}\|X(r)\|^{2} & =-\int_{r}^{t}\langle A X(s), X(s)\rangle d s-\int_{r}^{t} \int_{D} \xi(s) X(s) d s \\
& +\frac{1}{2} \int_{r}^{t}\|B(s)\|_{\mathscr{L}^{2}(U, H)}^{2}+\int_{r}^{t} X(s) B(s, X(s)) d W(s)
\end{aligned}
$$


everywhere on $\Omega^{\prime}$. By the definition of strong solution, we can assume that $X \in L^{\infty}(0, T ; H), A X \in$ $L^{2}\left(0, T ; V^{\prime}\right)$ and $j(X)+j^{*}(\xi) \in L^{1}((0, T) \times D)$, as well as that $B(\cdot, X) \in L^{2}\left(0, T ; \mathscr{L}^{2}(U, H)\right)$, everywhere on $\Omega^{\prime}$. Since $X \xi=j(X)+j^{*}(\xi)$, it follows that the process

$$
[0, T] \ni s \longmapsto \psi(s):=-\langle A X(s), X(s)\rangle-\int_{D} \xi(s) X(s)+\frac{1}{2}\|B(s, X(s))\|_{\mathscr{L}^{2}(U, H)}^{2}
$$

belongs to $L^{1}(0, T)$ everywhere on $\Omega^{\prime}$. Therefore, writing

$$
\frac{1}{2}\|X(t)\|^{2}-\frac{1}{2}\|X(r)\|^{2}=\int_{r}^{t} \phi(s) d s+\int_{r}^{t} X(s) B(s, X(s)) d W(s),
$$

since $\psi \in L^{1}(0, T)$ and the stochastic integral has continuous trajectories, we have, as $t \rightarrow r$,

$$
\|X(t)\|^{2}-\|X(r)\|^{2} \rightarrow 0,
$$

so that $\|X(t)\| \rightarrow\|X(r)\|$. Furthermore, $X(t) \rightarrow X(r)$ weakly in $H$ as $t \rightarrow r$ by Theorem 2.2 hence, since $H$ is uniformly convex, we conclude that $X(t) \rightarrow X(r)$ in $H$ (cf., e.g., [7, Proposition 3.32]).

\section{Existence and uniqueness}

We begin with a simple estimate that will be used several times.

Lemma 4.1. Let $F$ and $G$ be progressive process with values in $H$ and $\mathscr{L}^{2}(U, H)$, respectively, such that $F G$ is integrable with respect to $W$. For any numbers $p, \varepsilon>0$ and any stopping time $S$ one has

$$
\left\|((F G) \cdot W)_{S}^{*}\right\|_{L^{p}(\Omega)} \lesssim \varepsilon\left\|F_{S}^{*}\right\|_{L^{2 p}(\Omega)}^{2}+\frac{1}{\varepsilon}\left\|G \mathbb{1}_{\llbracket 0, S \rrbracket}\right\|_{L^{2 p}\left(\Omega ; L^{2}\left(0, T ; \mathscr{L}^{2}(U, H)\right)\right)}^{2}
$$

Proof. The BDG inequality asserts that

$$
\left\|((F G) \cdot W)_{S}^{*}\right\|_{L^{p}(\Omega)} \bar{\sim}\left\|[(F G) \cdot W,(F G) \cdot W]_{S}^{1 / 2}\right\|_{L^{p}(\Omega)},
$$

where, by the ideal property of Hilbert-Schmidt operators and Young's inequality,

$$
\begin{aligned}
{[(F G) \cdot W,(F G) \cdot W]_{S}^{1 / 2} } & =\left(\int_{0}^{S}\|F(t) G(t)\|_{\mathscr{L}^{2}(U, \mathbb{R})}^{2} d t\right)^{1 / 2} \\
& \leq\left(\int_{0}^{S}\|F(t)\|^{2}\|G(t)\|_{\mathscr{L}^{2}(U, H)}^{2} d t\right)^{1 / 2} \\
& \leq F_{S}^{*}\left(\int_{0}^{S}\|G(t)\|_{\mathscr{L}^{2}(U, H)}^{2} d t\right)^{1 / 2} \\
& \leq \varepsilon F_{S}^{* 2}+\frac{1}{\varepsilon} \int_{0}^{S}\|G(t)\|_{\mathscr{L}^{2}(U, H)}^{2} d t .
\end{aligned}
$$

Therefore, taking the $L^{p}(\Omega)$-(quasi)norm on both sides,

$$
\left\|((F G) \cdot W)_{S}^{*}\right\|_{L^{p}(\Omega)} \lesssim \varepsilon\left\|F_{S}^{*}\right\|_{L^{2 p}(\Omega)}^{2}+\frac{1}{\varepsilon}\left\|G \mathbb{1}_{\llbracket 0, S \rrbracket}\right\|_{L^{2 p}\left(\Omega ; L^{2}\left(0, T ; \mathscr{L}^{2}(U, H)\right)\right)}^{2}
$$

Let $(X, \xi)$ and $(Y, \eta) \in \mathscr{J}_{0}$ be strong solutions, in the sense of Definition 2.1 to the equation

$$
d X+A X d t+\beta(X) d t \ni B(\cdot, X) d W
$$

with initial conditions $X_{0}$ and $Y_{0}$, both elements of $L^{0}(\Omega, \mathscr{F}, \mathbb{P} ; H)$, respectively. Here and throughout this section we assume that $B$ is locally Lipschitz-continuous in the sense of assumption (B2).

Let us also introduce the sequence of stopping times $\left(T_{n}\right)_{n \in \mathbb{N}}$ defined as

$$
T_{n}:=\inf \left\{t \geq 0:\left\|X_{\Gamma}(t)\right\| \geq n \text { or }\left\|Y_{\Gamma}(t)\right\| \geq n\right\} \wedge T .
$$


Here and in the following, for any $\Gamma \in \mathscr{F}_{0}$, we shall denote multiplication by $\mathbb{1}_{\Gamma}$ by a subscript $\Gamma$. Even though the stopping times $T_{n}$ depend on $\Gamma$, we shall not indicate this explicitly to avoid making the notation too cumbersome.

The stopping times $T_{n}$ are well defined because, by definition of $\mathscr{J}_{0}, X$ and $Y$ have continuous paths with values in $H$. Moreover, $T_{n} \neq 0$ for sufficiently large $n$.

The estimate in the following lemma is an essential tool, from which, for instance, uniqueness and a local property of solutions will follow as easy corollaries.

Lemma 4.2. Let $\Gamma \in \mathscr{F}_{0}$ be such that $X_{0 \Gamma}, Y_{0 \Gamma} \in L^{2}\left(\Omega, \mathscr{F}_{0}, \mathbb{P} ; H\right)$. One has, for every $n \in \mathbb{N}$,

$$
\mathbb{E}\left(X_{\Gamma}-Y_{\Gamma}\right)_{T_{n}}^{* 2} \lesssim \mathbb{E}\left\|X_{0 \Gamma}-Y_{0 \Gamma}\right\|^{2}
$$

with implicit constant depending on $T$ and on the Lipschitz constant of $B$ in the ball in $H$ of radius $n$.

Proof. One has

$$
(X-Y)+\int_{0}^{t} A(X-Y) d s+\int_{0}^{t}(\xi-\eta) d s=X_{0}-Y_{0}+\int_{0}^{t}(B(X)-B(Y)) d W .
$$

We recall that, for any $\mathscr{F}_{0}$-measurable random variable $\zeta$ and any stochastically integrable process $K$, one has $\zeta(K \cdot W)=(\zeta K) \cdot W$. Therefore

$$
(X-Y)_{\Gamma}+\int_{0}^{t} A(X-Y)_{\Gamma} d s+\int_{0}^{t}(\xi-\eta)_{\Gamma} d s=\left(X_{0}-Y_{0}\right)_{\Gamma}+\int_{0}^{t}(B(X)-B(Y))_{\Gamma} d W
$$

The Itô formula of Theorem 3.2 yields

$$
\begin{aligned}
\| X_{\Gamma}- & \left.Y_{\Gamma} \|^{2}\left(t \wedge T_{n}\right)+2 \int_{0}^{t \wedge T_{n}}\left\langle A\left(X_{\Gamma}-Y_{\Gamma}\right), X_{\Gamma}-Y_{\Gamma}\right)\right\rangle d s+2 \int_{0}^{t \wedge T_{n}} \int_{D}((X-Y)(\xi-\eta))_{\Gamma} d s \\
= & \left\|X_{0 \Gamma}-Y_{0 \Gamma}\right\|^{2}+\int_{0}^{t \wedge T_{n}}\left\|(B(X)-B(Y))_{\Gamma}\right\|_{\mathscr{L}^{2}(U, H)}^{2} d s \\
& +2 \int_{0}^{t \wedge T_{n}}(X-Y)_{\Gamma}(B(X)-B(Y))_{\Gamma} d W,
\end{aligned}
$$

where (a) the second and term terms on the left-hand side are positive by monotonicity of $A$ and $\beta$, and by the assumption that $\xi \in \beta(X), \eta \in \beta(Y)$ a.e. in $\Omega \times(0, T) \times D$; (b) one has

$$
(B(X)-B(Y))_{\Gamma}=\mathbb{1}_{\Gamma}\left(B\left(X_{\Gamma}\right)-B\left(Y_{\Gamma}\right)\right)
$$

hence

$$
\mathbb{1}_{\llbracket 0, T_{n} \rrbracket}\left\|(B(X)-B(Y))_{\Gamma}\right\|_{\mathscr{L}^{2}(U, H)}^{2} \lesssim_{n} \mathbb{1}_{\llbracket 0, T_{n} \rrbracket} \mathbb{1}_{\Gamma}\left\|X_{\Gamma}-Y_{\Gamma}\right\| .
$$

Taking supremum in time and expectation,

$$
\begin{aligned}
\mathbb{E}\left(X_{\Gamma}^{T_{n}}-Y_{\Gamma}^{T_{n}}\right)_{t}^{* 2} \lesssim & \mathbb{E}\left\|X_{0 \Gamma}-Y_{0 \Gamma}\right\|^{2}+\int_{0}^{t} \mathbb{E}\left(X_{\Gamma}^{T_{n}}-Y_{\Gamma}^{T_{n}}\right)_{s}^{* 2} d s \\
& +\mathbb{E} \sup _{s \leq t} \int_{0}^{s \wedge T_{n}}(X-Y)_{\Gamma}(B(X)-B(Y))_{\Gamma} d W
\end{aligned}
$$

where, by Lemma 4.1] the last term on the right-hand side is bounded by

$$
\begin{gathered}
\varepsilon \mathbb{E}\left(X_{\Gamma}^{T_{n}}-Y_{\Gamma}^{T_{n}}\right)_{t}^{* 2}+N(\varepsilon) \mathbb{E} \int_{0}^{t \wedge T_{n}}\left\|(B(X)-B(Y))_{\Gamma}\right\|_{\mathscr{L}^{2}(U, H)}^{2} d s \\
\leq \varepsilon \mathbb{E}\left(X_{\Gamma}^{T_{n}}-Y_{\Gamma}^{T_{n}}\right)_{t}^{* 2}+N(\varepsilon, n) \int_{0}^{t} \mathbb{E}\left(X_{\Gamma}^{T_{n}}-Y_{\Gamma}^{T_{n}}\right)_{s}^{* 2} d s .
\end{gathered}
$$

Choosing $\varepsilon$ small enough, it follows by Gronwall's inequality that

$$
\mathbb{E}\left(X_{\Gamma}-Y_{\Gamma}\right)_{T_{n}}^{* 2}=\mathbb{E}\left(X_{\Gamma}^{T_{n}}-Y_{\Gamma}^{T_{n}}\right)_{T}^{* 2} \lesssim \mathbb{E}\left\|X_{0 \Gamma}-Y_{0 \Gamma}\right\|^{2},
$$

with an implicit constant that depends on $T$ and on the Lipschitz constant of $B$ on the ball in $H$ of radius $n$. 
Corollary 4.3. Uniqueness of strong solutions in $\mathscr{J}_{0}$ holds for (1.1).

Proof. Let $(X, \xi),(Y, \eta) \in \mathscr{J}_{0}$ be strong solutions to (1.1). For any $\Gamma \in \mathscr{F}_{0}$ such that $X_{0 \Gamma} \in L^{2}(\Omega ; H)$ the previous lemma yields $X_{\Gamma}^{T_{n}}=Y_{\Gamma}^{T_{n}}$ for all $n \in \mathbb{N}$, hence $X_{\Gamma}=Y_{\Gamma}$. Writing

$$
\Omega=\bigcup_{k \in \mathbb{N}} \Omega_{k}, \quad \Omega_{k}:=\left\{\omega \in \Omega:\left\|X_{0}(\omega)\right\| \leq k\right\}
$$

and choosing $\Gamma$ as $\Omega_{k}$, it follows that $X \mathbb{1}_{\Omega_{k}}=Y \mathbb{1}_{\Omega_{k}}$ for all $k$, hence $X=Y$. By comparison, $\xi=\eta$ a.e. in $\Omega \times(0, T) \times D$.

Remark 4.4. To prove the corollary, by inspection of the proof of Lemma 4.2 it is evident that one may directly take $\Gamma=\Omega$, as in this case $X_{0}-Y_{0}=0$, whose second moment is obviously finite. This immediately implies $X^{T_{n}}=Y^{T_{n}}$ for all $n \in \mathbb{N}$, hence $X=Y$.

Corollary 4.5. Let $\Gamma \in \mathscr{F}_{0}$. If $X_{0 \Gamma}=Y_{0 \Gamma}$, then $X_{\Gamma}=Y_{\Gamma}$, and $\xi_{\Gamma}=\eta_{\Gamma}$ a.e. in $\Omega \times(0, T) \times D$.

Proof. Write $\Omega=\bigcup_{k \in \mathbb{N}} \Omega_{k}$, where

$$
\Omega_{k}:=\left\{\omega \in \Omega:\left\|X_{0}(\omega)\right\| \leq k\right\} \cap\left\{\omega \in \Omega:\left\|Y_{0}(\omega)\right\| \leq k\right\} .
$$

Then $X_{0} \mathbb{1}_{\Gamma \cap \Omega_{k}}, Y_{0} \mathbb{1}_{\Gamma \cap \Omega_{k}} \in L^{2}(\Omega ; H)$, and Lemma 4.2 implies that $X_{\Gamma \cap \Omega_{k}}=Y_{\Gamma \cap \Omega_{k}}$ for all $k \in \mathbb{N}$, hence $X_{\Gamma}=Y_{\Gamma}$, as well as, again by comparison, $\xi_{\Gamma}=\eta_{\Gamma}$ a.e. in $\Omega \times(0, T) \times D$.

Now that uniqueness is cleared, we turn to the question of existence of strong solutions. For this we need some preparations. For $R>0$, let us consider the truncation operator $\sigma_{R}: H \rightarrow H$ defined as

$$
\sigma_{R}: x \longmapsto \begin{cases}x, & \|x\| \leq R, \\ R x /\|x\|, & \|x\|>R .\end{cases}
$$

We shall then define

$$
\begin{aligned}
B_{R}: \Omega \times[0, T] \times H & \longrightarrow \mathscr{L}^{2}(U, H) \\
(\omega, t, x) & \longmapsto B\left(\omega, t, \sigma_{R}(x)\right) .
\end{aligned}
$$

Let us check that $B_{R}$ is Lipschitz-continuous for every $R>0$. The progressive measurability of $B_{R}$ follows from the one of $B$ and the fact that $\sigma_{R}: H \rightarrow H$ is (Lipschitz) continuous. Moreover, since $\sigma_{R}$ is 1-Lipschitz continuous, thanks to the local Lipschitz continuity and the linear growth of $B$, for every $\omega \in \Omega, t \in[0, T]$ and $x, y \in H$ one has

$$
\left\|B_{R}(\omega, t, x)-B_{R}(\omega, t, y)\right\|_{\mathscr{L}^{2}(U, H)} \leq N_{R}\left\|\sigma_{R}(x)-\sigma_{R}(y)\right\| \leq N_{R}\|x-y\|
$$

as well as

$$
\left\|B_{R}(\omega, t, x)\right\|_{\mathscr{L}^{2}(U, H)} \leq N\left(1+\left\|\sigma_{R}(x)\right\|\right) \leq N(1+\|x\|) .
$$

Thanks to Theorems 2.2 and 3.3. as well as Lemma 4.2 the equation

$$
d X_{n}+A X_{n} d t+\beta\left(X_{n}\right) d t=B_{n}\left(X_{n}\right) d W, \quad X_{n}(0)=X_{0},
$$

admits a strong solution $\left(X_{n}, \xi_{n}\right)$, which belongs to $\mathscr{J}_{2}$ and is unique in $\mathscr{J}_{0}$, for every $n \in \mathbb{N} 1$ Moreover, by the strong continuity of the paths of $X_{n}$, one can define the increasing sequence of stopping times $\left(\tau_{n}\right)_{n \in \mathbb{N}}$ by

$$
\tau_{n}:=\inf \left\{t \in[0, T]:\left\|X_{n}(t)\right\| \geq n\right\},
$$

as well as the stopping time

$$
\tau:=\lim _{n \rightarrow \infty} \tau_{n}=\sup _{n \in \mathbb{N}} \tau_{n}
$$

As first step we show that the sequence of processes $\left(X_{n}, \xi_{n}\right)$ satisfies a sort of consistency condition.

\footnotetext{
${ }^{1}$ Note that Theorem 2.2 only shows that $\left(X_{n}, \xi_{n}\right)$ is unique in $\mathscr{J}_{2}$, while Lemma 4.2 yields uniqueness in the larger space $\mathscr{J}_{0}$.
} 
Lemma 4.6. One has $X_{n+1}^{\tau_{n}}=X_{n}^{\tau_{n}}$ for all $n \in \mathbb{N}$, as well as $\xi_{n} \mathbb{1}_{\llbracket 0, \tau_{n} \rrbracket}=\xi_{n+1} \mathbb{1}_{\llbracket 0, \tau_{n} \rrbracket}$ in $L^{0}(\Omega \times(0, T) \times$ $D)$.

Proof. Itô's formula yields, in view of the monotonicity of $A$ and $\beta$,

$$
\begin{aligned}
\left\|X_{n+1}-X_{n}\right\|^{2}\left(t \wedge \tau_{n}\right) \lesssim & \int_{0}^{t \wedge \tau_{n}}\left(X_{n+1}-X_{n}\right)\left(B_{n+1}\left(X_{n+1}\right)-B_{n}\left(X_{n}\right)\right)(s) d W(s) \\
& +\int_{0}^{t \wedge \tau_{n}}\left\|B_{n+1}\left(X_{n+1}(s)\right)-B_{n}\left(X_{n}(s)\right)\right\|_{\mathscr{L}^{2}(U, H)}^{2} d s .
\end{aligned}
$$

Note that $B_{n+1}=B_{n}$ on the ball of radius $n$ in $H$, hence $B_{n}\left(X_{n}\right)=B_{n+1}\left(X_{n}\right)$ on $\llbracket 0, \tau_{n} \rrbracket$. Therefore, since $B_{n+1}$ is Lipschitz continuous,

$$
\begin{aligned}
\mathbb{E}\left(X_{n+1}^{\tau_{n}}-X_{n}^{\tau_{n}}\right)_{t}^{* 2} \lesssim & \mathbb{E}\left(\left(\left(X_{n+1}-X_{n}\right)\left(B_{n+1}\left(X_{n+1}\right)-B_{n+1}\left(X_{n}\right)\right)\right) \cdot W\right)_{t \wedge \tau_{n}}^{*} \\
& +\int_{0}^{t} \mathbb{E}\left(X_{n+1}^{\tau_{n}}-X_{n}^{\tau_{n}}\right)_{s}^{* 2} d s,
\end{aligned}
$$

where the first term on the right-hand side can be estimated, thanks to the BDG inequality and the ideal property of Hilbert-Schmidt operators, by

$$
\begin{aligned}
& \mathbb{E}\left(\int_{0}^{t \wedge \tau_{n}}\right.\left.\left\|X_{n+1}-X_{n}\right\|^{2}(s)\left\|B_{n+1}\left(X_{n+1}(s)\right)-B_{n+1}\left(X_{n}(s)\right)\right\|_{\mathscr{L}^{2}(U, H)}^{2} d s\right)^{1 / 2} \\
& \lesssim_{n} \mathbb{E}\left(X_{n+1}^{\tau_{n}}-X_{n}^{\tau_{n}}\right)_{t}^{*}\left(\int_{0}^{t}\left\|X_{n+1}^{\tau_{n}}-X_{n}^{\tau_{n}}\right\|^{2}(s)\right)^{1 / 2} \\
& \quad{ }_{n} \varepsilon \mathbb{E}\left(X_{n+1}^{\tau_{n}}-X_{n}^{\tau_{n}}\right)_{t}^{* 2}+\frac{1}{\varepsilon} \int_{0}^{t} \mathbb{E}\left(X_{n+1}^{\tau_{n}}-X_{n}^{\tau_{n}}\right)_{s}^{* 2} d s .
\end{aligned}
$$

Choosing $\varepsilon$ small enough, Gronwall's inequality implies

$$
\mathbb{E}\left(X_{n+1}^{\tau_{n}}-X_{n}^{\tau_{n}}\right)_{t}^{* 2}=0
$$

for all $t \leq T$, hence $X_{n+1}^{\tau_{n}}=X_{n}^{\tau_{n}}$. The first claim is thus proved.

In order to prove the second claim, note that it holds

$$
\begin{gathered}
X_{n+1}^{\tau_{n}}(t)+\int_{0}^{t \wedge \tau_{n}} A X_{n+1} d s+\int_{0}^{t \wedge \tau_{n}} \xi_{n+1} d s=X_{0}+\int_{0}^{t \wedge \tau_{n}} B_{n+1}\left(X_{n+1}\right) d W, \\
X_{n}^{\tau_{n}}(t)+\int_{0}^{t \wedge \tau_{n}} A X_{n} d s+\int_{0}^{t \wedge \tau_{n}} \xi_{n} d s=X_{0}+\int_{0}^{t \wedge \tau_{n}} B_{n}\left(X_{n}\right) d W,
\end{gathered}
$$

where $B_{n}\left(X_{n}\right)$ on the right-hand side of the second identity can be replaced by $B_{n+1}\left(X_{n+1}\right)$ because the paths of $X_{n+1}^{\tau_{n}}$ remain within a ball of radius $n$ in $H$ and $X_{n+1}^{\tau_{n}}=X_{n}^{\tau_{n}}$. This identity also yields, by comparison,

$$
\int_{0}^{t} \xi_{n+1} \mathbb{1}_{\llbracket 0, \tau_{n} \rrbracket} d s=\int_{0}^{t \wedge \tau_{n}} \xi_{n+1} d s=\int_{0}^{t \wedge \tau_{n}} \xi_{n} d s=\int_{0}^{t} \xi_{n} \mathbb{1}_{\llbracket 0, \tau_{n} \rrbracket} d s
$$

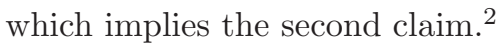

The lemma implies that one can define processes $X$ and $\xi$ on $\llbracket 0, \tau \rrbracket$ by the prescriptions $X:=X_{n}$ and $\xi:=\xi_{n}$ on $\llbracket 0, \tau_{n} \rrbracket$ for all $n \in \mathbb{N}$, or equivalently (but perhaps less tellingly), as $X=\lim _{n \rightarrow \infty} X_{n}$ and $\xi=\lim _{n \rightarrow \infty} \xi_{n}$.

We are now going to show that the linear growth assumption on $B$ implies that $\tau=T$. We shall first establish a priori estimates for the solution to equation (4.1).

\footnotetext{
${ }^{2}$ The argument in fact proves the following slightly stronger statement: setting $\Xi_{n}:=\int_{0}^{*} \xi_{n} d s$, the processes $\Xi_{n+1}^{\tau_{n}}$ and $\Xi_{n}^{\tau_{n}}$ are indistinguishable for all $n$.
} 
Lemma 4.7. There exists a constant $N>0$, independent of $n$, such that

$$
\mathbb{E}\left\|X_{n}\right\|_{C([0, T] ; H)}^{2}+\mathbb{E}\left\|X_{n}\right\|_{L^{2}(0, T ; V)}^{2}+\mathbb{E}\left\|\xi_{n} X_{n}\right\|_{L^{1}((0, T) \times D)}<N\left(1+\mathbb{E}\left\|X_{0}\right\|^{2}\right) .
$$

Proof. The Itô formula of Theorem 3.2 yields

$$
\begin{aligned}
& \left\|X_{n}(t)\right\|^{2}+2 \int_{0}^{t}\left\langle A X_{n}(s), X_{n}(s)\right\rangle d s+2 \int_{0}^{t} \int_{D} \xi_{n}(s) X_{n}(s) d x d s \\
& =\left\|X_{0}\right\|^{2}+\int_{0}^{t}\left\|B_{n}\left(s, X_{n}(s)\right)\right\|_{\mathscr{L}^{2}(U, H)}^{2} d s+2 \int_{0}^{t} X_{n}(s) B_{n}\left(s, X_{n}(s)\right) d W(s)
\end{aligned}
$$

where, recalling that $B_{n}=B\left(\cdot, \cdot, \sigma_{n}(\cdot)\right)$ and $\sigma_{n}$ is a contraction in $H$, and that $B$ grows at most linearly,

$$
\int_{0}^{t}\left\|B_{n}\left(s, X_{n}(s)\right)\right\|_{\mathscr{L}^{2}(U, H)}^{2} \lesssim T+\int_{0}^{T}\left\|X_{n}(s)\right\|^{2} d s .
$$

Denoting the stochastic integral on the right-hand side by $M_{n}$, taking supremum in time and expectation we get, by the coercivity of $A$,

$$
\begin{gathered}
\mathbb{E}\left\|X_{n}\right\|_{C([0, T] ; H)}^{2}+\mathbb{E}\left\|X_{n}\right\|_{L^{2}(0, T ; V)}^{2}+\mathbb{E}\left\|\xi_{n} X_{n}\right\|_{L^{1}((0, T) \times D)} \\
\lesssim 1+\mathbb{E}\left\|X_{0}\right\|^{2}+\mathbb{E} \int_{0}^{T}\left\|X_{n}(s)\right\|^{2} d s+\mathbb{E} M_{T}^{* 2}
\end{gathered}
$$

where the implicit constant depends on T. By Lemma 4.1 we have, for any $\varepsilon>0$,

$$
\mathbb{E} M_{T}^{* 2} \lesssim \varepsilon \mathbb{E}\left\|X_{n}\right\|_{C([0, T] ; H)}^{2}+N(\varepsilon) \mathbb{E} \int_{0}^{T}\left\|X_{n}(s)\right\|^{2} d s,
$$

therefore, choosing $\varepsilon$ sufficiently small,

$$
\begin{aligned}
\mathbb{E}\left\|X_{n}\right\|_{C([0, T] ; H)}^{2}+\mathbb{E}\left\|X_{n}\right\|_{L^{2}(0, T ; V)}^{2}+\mathbb{E}\left\|\xi_{n} X_{n}\right\|_{L^{1}((0, T) \times D)} \\
\quad \lesssim 1+\mathbb{E}\left\|X_{0}\right\|^{2}+\mathbb{E} \int_{0}^{T}\left\|X_{n}(s)\right\|^{2} d s
\end{aligned}
$$

Since this inequality holds also with $T$ replaced by any $t \in] 0, T]$, we also have

$$
\mathbb{E}\left\|X_{n}\right\|_{C([0, t] ; H)}^{2} \lesssim 1+\mathbb{E}\left\|X_{0}\right\|^{2}+\int_{0}^{T} \mathbb{E}\left\|X_{n}\right\|_{C([0, s] ; H)}^{2} d s,
$$

hence, by Gronwall's inequality,

$$
\mathbb{E}\left\|X_{n}\right\|_{C([0, T] ; H)}^{2} \lesssim 1+\mathbb{E}\left\|X_{0}\right\|^{2}
$$

with implicit constant depending on $T$. Since $C([0, T] ; H)$ is continuously embedded in $L^{2}(0, T ; H)$, one easily deduces

$$
\mathbb{E}\left\|X_{n}\right\|_{C([0, T] ; H)}^{2}+\mathbb{E}\left\|X_{n}\right\|_{L^{2}(0, T ; V)}^{2}+\mathbb{E}\left\|\xi_{n} X_{n}\right\|_{L^{1}((0, T) \times D)} \lesssim 1+\mathbb{E}\left\|X_{0}\right\|^{2}
$$

Lemma 4.8. One has

$$
\mathbb{P}\left(\limsup _{n \rightarrow \infty}\left\{\tau_{n} \leq T\right\}\right)=0
$$

In particular, $\tau=T$.

Proof. By Markov's inequality and the previous lemma,

$$
\mathbb{P}\left(\left\|X_{n}\right\|_{C([0, T] ; H)} \geq n\right) \leq \frac{1}{n^{2}} \mathbb{E}\left\|X_{n}\right\|_{C([0, T] ; H)}^{2} \lesssim \frac{1}{n^{2}}\left(1+\mathbb{E}\left\|X_{0}\right\|^{2}\right)
$$


Since the event $\left\{\left\|X_{n}\right\|_{C([0, T] ; H)} \geq n\right\}$ coincides with $\left\{\tau_{n} \leq T\right\}$, one has

$$
\sum_{n=1}^{\infty} \mathbb{P}\left(\tau_{n} \leq T\right)<\infty
$$

thus also, by the Borel-Cantelli lemma,

$$
\mathbb{P}\left(\bigcap_{n \in \mathbb{N}} \bigcup_{k \geq n}\left\{\tau_{k} \leq T\right\}\right)=0 .
$$

In other words, the sequence $\left(\tau_{n}\right)$ is ultimately constant: for each $\omega$ in a subset of $\Omega$ of $\mathbb{P}$-measure one, there exists $m=m(\omega)$ such that $\tau_{n}(\omega)=T$ for all $n>m$. In particular, $\tau=T \mathbb{P}$-almost surely.

This lemma implies that the processes $X$ and $\xi$ defined immediately after the proof of Lemma 4.6 are indeed defined on the whole interval $[0, T]$.

We can now prove the first existence result.

Theorem 4.9. Assume that $X_{0} \in L^{2}\left(\Omega, \mathscr{F}_{0}, \mathbb{P} ; H\right)$. Then equation (1.1) admits a unique strong solution, which belongs to $\mathscr{J}_{2}$.

Proof. Uniqueness of strong solutions is proved, in more generality, by Corollary 4.3 Let us prove existence. By stopping at $\tau_{n}$, one has

$$
X_{n}^{\tau_{n}}(t)+\int_{0}^{t \wedge \tau_{n}} A X_{n}(s) d s+\int_{0}^{t \wedge \tau_{n}} \xi_{n}(s) d s=X_{0}+\int_{0}^{t \wedge \tau_{n}} B_{n}\left(X_{n}(s)\right) d s,
$$

where, by definition of $X, X_{n}^{\tau_{n}}=X^{\tau_{n}}$, as well as, by definition of $B_{n}$,

$$
\int_{0}^{t \wedge \tau_{n}} B_{n}\left(X_{n}(s)\right) d s=\int_{0}^{t \wedge \tau_{n}} B(X(s)) d s .
$$

Similarly, by definition of $\xi$ it follows that

$$
\int_{0}^{t \wedge \tau_{n}} \xi_{n}(s) d s=\int_{0}^{t \wedge \tau_{n}} \xi(s) d s
$$

hence that

$$
X^{\tau_{n}}(t)+\int_{0}^{t \wedge \tau_{n}} A X(s) d s+\int_{0}^{t \wedge \tau_{n}} \xi(s) d s=X_{0}+\int_{0}^{t \wedge \tau_{n}} B(X(s)) d s .
$$

Since this identity holds for all $n \in \mathbb{N}$ and $\tau_{n} \rightarrow T$ as $n \rightarrow \infty$, we infer that

$$
X(t)+\int_{0}^{t} A X(s) d s+\int_{0}^{t} \xi(s) d s=X_{0}+\int_{0}^{t} B(X(s)) d s
$$

for all $t \in[0, T] \mathbb{P}$-a.s.. Moreover, for every $n \in \mathbb{N}, \xi_{n} \in \beta\left(X_{n}\right)$ a.e. in $\Omega \times(0, T) \times D$, hence $\xi_{n} \mathbb{1}_{\llbracket 0, \tau_{n} \rrbracket} \in \beta\left(X_{n}\right) \mathbb{1}_{\llbracket 0, \tau_{n} \rrbracket}$, thus also $\xi \mathbb{1}_{\llbracket 0, \tau_{n} \rrbracket} \in \beta(X) \mathbb{1}_{\llbracket 0, \tau_{n} \rrbracket}$ a.e. in $\Omega \times(0, T) \times D$. Recalling that $\tau_{n} \rightarrow T$ as $n \rightarrow \infty$, this in turn implies $\xi \in \beta(X)$ a.e. in $\Omega \times(0, T) \times D$.

Moreover, since $(X, \xi)$ is the almost sure limit of $\left(X_{n}, \xi_{n}\right)$, we immediately infer that $X$ and $\xi$ are predictable $H$-valued and $L^{1}(D)$-valued processes, respectively. The a priori estimates of Lemma 4.7 and Fatou's lemma then yield

$$
X \in L^{2}(\Omega ; C([0, T] ; H)) \cap L^{2}\left(\Omega ; L^{2}(0, T ; V)\right), \quad \xi \in L^{1}(\Omega \times(0, T) \times D) .
$$

Similarly, $\xi_{n} \in \beta\left(X_{n}\right)$ implies $X_{n} \xi_{n}=j\left(X_{n}\right)+j^{*}\left(\xi_{n}\right)$, hence

$$
\mathbb{E} \int_{0}^{T} \int_{D}\left(j\left(X_{n}\right)+j^{*}\left(\xi_{n}\right)\right) \lesssim 1+\mathbb{E}\left\|X_{0}\right\|^{2}
$$

for all $n \in \mathbb{N}$, and again by Fatou's lemma, as well as by the lower-semicontinuity of convex integrals, one obtains

$$
j(X)+j^{*}(\xi) \in L^{1}\left(\Omega ; L^{1}\left(0, T ; L^{1}(D)\right)\right) .
$$

We have thus proved that $(X, \xi) \in \mathscr{J}_{2}$, so the proof is completed. 
The second existence result, which allows $X_{0}$ to be merely $\mathscr{F}_{0}$-measurable, follows by a further "gluing" procedure.

Theorem 4.10. Assume that $X_{0} \in L^{0}\left(\Omega, \mathscr{F}_{0}, \mathbb{P} ; H\right)$. Then equation (1.1) admits a unique strong solution.

Proof. Uniqueness of strong solutions has already been proved in Corollary 4.3. It is hence enough to prove existence. Let us define the sequence $\left(\Gamma_{n}\right)_{n \in \mathbb{N}}$ of elements of $\mathscr{F}_{0}$ as

$$
\Gamma_{n}:=\left\{\omega \in \Omega:\left\|X_{0}\right\| \leq n\right\} .
$$

It is evident that $\left(\Gamma_{n}\right)$ is a sequence increasing to $\Omega$, and that $X_{0 \Gamma_{n}}=X_{0} \mathbb{1}_{\Gamma_{n}} \in L^{2}(\Omega ; H)$. Therefore, by the previous theorem, for each $n \in \mathbb{N}$ there exists a unique strong solution $\left(X_{n}, \xi_{n}\right)$ to (1.1) with initial condition $X_{0 \Gamma_{n}}$. By the local property of solutions established in Corollary 4.5, we have that $X_{n+1} \mathbb{1}_{\Gamma_{n}}$ and $X_{n} \mathbb{1}_{\Gamma_{n}}$ are indistinguishable, and $\xi_{n+1} \mathbb{1}_{\Gamma_{n}}=\xi_{n} \mathbb{1}_{\Gamma_{n}}$ a.e. in $\Omega \times(0, T) \times D$. Since $\left(\Gamma_{n}\right)$ is increasing, it makes sense to define the processes $X$ and $\xi$ by

$$
X \mathbb{1}_{\Gamma_{n}}=X_{n} \mathbb{1}_{\Gamma_{n}}, \quad \xi \mathbb{1}_{\Gamma_{n}}=\xi_{n} \mathbb{1}_{\Gamma_{n}}
$$

for all $n \in \mathbb{N}$. This amounts to saying that $X$ and $\xi$ are the $\mathbb{P}$-a.s. limits of $X_{n}$ and $\xi_{n}$, respectively, which immediately implies that $X$ and $\xi$ are predictable processes with values in $H$ and $L^{1}(D)$, respectively. Moreover, by construction, we also have

$$
X \in L^{0}\left(\Omega ; C([0, T] ; H) \cap L^{2}(0, T ; V)\right), \quad \xi \in L^{0}\left(\Omega ; L^{1}\left(0, T ; L^{1}(D)\right)\right)
$$

In fact, writing $E:=C([0, T] ; H) \cap L^{2}(0, T ; V)$ for compactness of notation, by the previous theorem we have $X_{n} \in L^{2}(\Omega ; E)$ and $\xi \in L^{1}(\Omega \times(0, T) \times D)$, and for any arbitrary but fixed $\omega$ in a subset of $\Omega$ of probability one, there exists $n=n(\omega)$ such that $(X(\omega), \xi(\omega))=\left(X_{n}(\omega), \xi_{n}(\omega)\right) \in E \times L^{1}((0, T) \times D)$. Furthermore, since $\xi_{n} \in \beta\left(X_{n}\right)$ a.e. for all $n \in \mathbb{N}$, it is easy to see that

$$
\xi \mathbb{1}_{\Gamma_{n}}=\xi_{n} \mathbb{1}_{\Gamma_{n}} \in \beta\left(X_{n}\right) \mathbb{1}_{\Gamma_{n}}=\beta\left(X_{n} \mathbb{1}_{\Gamma_{n}}\right) \mathbb{1}_{\Gamma_{n}}=\beta(X) \mathbb{1}_{\Gamma_{n}}
$$

for all $n \in \mathbb{N}$, so that $\xi \in \beta(X)$ a.e. because $\Gamma_{n} \uparrow \Omega$. Similarly,

$$
j\left(X_{n}\right) \mathbb{1}_{\Gamma_{n}}=j\left(\mathbb{1}_{\Gamma_{n}} X_{n}\right) \mathbb{1}_{\Gamma_{n}}=j(X) \mathbb{1}_{\Gamma_{n}}
$$

as well as, by the same reasoning, $j^{*}\left(\xi_{n}\right) \mathbb{1}_{\Gamma_{n}}=j^{*}(\xi) \mathbb{1}_{\Gamma_{n}}$. Since, by the previous theorem, $j\left(X_{n}\right)+$ $j^{*}\left(\xi_{n}\right) \in L^{1}\left(\Omega ; L^{1}((0, T) \times D)\right.$ for all $n \in \mathbb{N}$, it follows that

$$
\left(j(X)+j^{*}(\xi)\right) \mathbb{1}_{\Gamma_{n}} \in L^{1}\left(\Omega ; L^{1}((0, T) \times D) \quad \forall n \in \mathbb{N},\right.
$$

hence $j(X)+j^{*}(\xi) \in L^{0}\left(\Omega ; L^{1}((0, T) \times D)\right.$.

\section{Moment estimates and dependence on the initial datum}

We are now going to show that the integrability of the solution is determined by the integrability of the initial condition.

Theorem 5.1. Let $p \geq 0$. If $X_{0} \in L^{p}\left(\Omega, \mathscr{F}_{0}, \mathbb{P} ; H\right)$, then the unique strong solution to equation (1.1) belongs to $\mathscr{J}_{p}$.

Proof. Itô's formula yields

$$
\begin{aligned}
\|X(t)\|^{2} & +2 \int_{0}^{t}\langle A X(s), X(s)\rangle d s+2 \int_{0}^{t} \int_{D} \xi(s) X(s) d x d s \\
& =\left\|X_{0}\right\|^{2}+\int_{0}^{t}\|B(s, X(s))\|_{\mathscr{L}^{2}(U, H)}^{2} d s+2 \int_{0}^{t} X(s) B(s, X(s)) d W(s) .
\end{aligned}
$$


For any $\alpha>0$, it follows by the integration-by-parts formula that

$$
\begin{aligned}
& e^{-2 \alpha t}\|X(t)\|^{2}+2 \alpha \int_{0}^{t} e^{-2 \alpha s}\|X(s)\|^{2} d s+2 \int_{0}^{t} e^{-2 \alpha s}\langle A X(s), X(s)\rangle d s \\
& +2 \int_{0}^{t} \int_{D} e^{-2 \alpha s} \xi(s) X(s) d x d s \\
& \quad=\left\|X_{0}\right\|^{2}+\int_{0}^{t} e^{-2 \alpha s}\|B(s, X(s))\|_{\mathscr{L}^{2}(U, H)}^{2} d s+2 \int_{0}^{t} e^{-2 \alpha s} X(s) B(s, X(s)) d W(s) .
\end{aligned}
$$

Let $M$ denote the stochastic integral on the right-hand side, and $Y(t):=e^{-\alpha t} X(t)$. Since $X$ has continuous paths in $H$, one can introduce the sequence of stopping times $\left(T_{n}\right)_{n \in \mathbb{N}}$, increasing to $T$, as

$$
T_{n}:=\inf \{t \geq 0:\|X(t)\| \geq n\} \wedge T \text {. }
$$

It follows by the local Lipschitz-continuity property of $B$ that

$$
\begin{gathered}
\left\|Y^{T_{n}}(t)\right\|^{2}+2 \alpha \int_{0}^{t \wedge T_{n}}\|Y(s)\|^{2} d s+2 C \int_{0}^{t \wedge T_{n}}\|Y(s)\|_{V}^{2} d s+2 \int_{0}^{t \wedge T_{n}} \int_{D} e^{-2 \alpha s} \xi(s) X(s) d x d s \\
\leq\left\|X_{0}\right\|^{2}+\int_{0}^{t \wedge T_{n}} e^{-2 \alpha s}\left\|B_{n}(s, X(s))\right\|_{\mathscr{L}^{2}(U, H)}^{2} d s+2 M^{T_{n}}(t) .
\end{gathered}
$$

Recalling that $B_{n}=B\left(\cdot, \cdot, \sigma_{n}(\cdot)\right)$ and $\sigma_{n}$ is a contraction in $H$, and that $B$ grows at most linearly, one has

$$
e^{-2 \alpha s}\left\|B_{n}(s, X(s))\right\|_{\mathscr{L}^{2}(U, H)}^{2} \lesssim e^{-2 \alpha s}+\|Y(s)\|^{2},
$$

hence

$$
\int_{0}^{t \wedge T_{n}} e^{-2 \alpha s}\left\|B_{n}(s, X(s))\right\|_{\mathscr{L}^{2}(U, H)}^{2} d s \lesssim \frac{1}{2 \alpha}+\int_{0}^{t \wedge T_{n}}\|Y(s)\|^{2} d s .
$$

Taking supremum in time and the $L^{p / 2}(\Omega)$-(quasi)norm, recalling the BDG inequality and the fact that $e^{-\alpha t} \xi_{n} X_{n} \geq e^{-\alpha T} \xi_{n} X_{n}$, we are left with

$$
\begin{aligned}
& \left\|Y_{T_{n}}^{*}\right\|_{L^{p}(\Omega)}^{2}+\alpha\left\|Y \mathbb{1}_{\llbracket 0, T_{n} \rrbracket}\right\|_{L^{p}\left(\Omega ; L^{2}(0, T ; H)\right)}^{2}+\left\|Y \mathbb{1}_{\llbracket 0, T_{n} \rrbracket}\right\|_{L^{p}\left(\Omega ; L^{2}(0, T ; V)\right)}^{2} \\
& \quad+e^{-\alpha T}\left\|\xi X \mathbb{1}_{\llbracket 0, T_{n} \rrbracket}\right\|_{L^{p / 2}\left(\Omega ; L^{1}((0, T) \times D)\right)} \\
& \quad \lesssim\left\|X_{0}\right\|_{L^{p}(\Omega ; H)}^{2}+\frac{1}{2 \alpha}+\left\|Y \mathbb{1}_{\llbracket 0, T_{n} \rrbracket}\right\|_{L^{p}\left(\Omega ; L^{2}(0, T ; H)\right)}^{2}+\left\|[M, M]_{T_{n}}^{1 / 2}\right\|_{L^{p / 2}(\Omega)} .
\end{aligned}
$$

Lemma 4.1 and (5.1) yield

$$
[M, M]_{T_{n}}^{1 / 2} \lesssim \varepsilon Y_{T_{n}}^{* 2}+\frac{1}{\varepsilon}\left(\frac{1}{2 \alpha}+\left\|Y \mathbb{1}_{\llbracket 0, T_{n} \rrbracket}\right\|_{L^{2}(0, T ; H)}^{2}\right)
$$

hence

$$
\left\|[M, M]_{T_{n}}^{1 / 2}\right\|_{L^{p / 2}(\Omega)} \lesssim \varepsilon\left\|Y_{T_{n}}^{*}\right\|_{L^{p}(\Omega)}^{2}+\frac{1}{\varepsilon}\left\|Y \mathbb{1}_{\llbracket 0, T_{n} \rrbracket}\right\|_{L^{p}\left(\Omega ; L^{2}(0, T ; H)\right)}^{2}+\frac{1}{2 \alpha \varepsilon},
$$

where the implicit constant is independent of $\alpha$ and of an arbitrary $\varepsilon>0$ to be chosen later. We thus have

$$
\begin{aligned}
& \left\|Y_{T_{n}}^{*}\right\|_{L^{p}(\Omega)}^{2}+\alpha\left\|Y \mathbb{1}_{\llbracket 0, T_{n} \rrbracket}\right\|_{L^{p}\left(\Omega ; L^{2}(0, T ; H)\right)}^{2}+\left\|Y \mathbb{1}_{\llbracket 0, T_{n} \rrbracket}\right\|_{L^{p}\left(\Omega ; L^{2}(0, T ; V)\right)}^{2} \\
& \quad+e^{-\alpha T}\left\|\xi X \mathbb{1}_{\llbracket 0, T_{n} \rrbracket}\right\|_{L^{p / 2}\left(\Omega ; L^{1}((0, T) \times D)\right)} \\
& \quad \lesssim\left\|X_{0}\right\|_{L^{p}(\Omega ; H)}^{2}+\varepsilon\left\|\left(Y^{T_{n}}\right)_{T}^{*}\right\|_{L^{p}(\Omega)}^{2}+(1+1 / \varepsilon)\left\|Y \mathbb{1}_{\llbracket 0, T_{n} \rrbracket}\right\|_{L^{p}\left(\Omega ; L^{2}(0, T ; H)\right)}^{2}+\frac{1}{2 \alpha}(1+1 / \varepsilon) .
\end{aligned}
$$

Since the implicit constant is independent of $\alpha$ and $\varepsilon$, one can take $\varepsilon$ small enough and $\alpha$ large enough so that

$$
\left\|Y_{T_{n}}^{*}\right\|_{L^{p}(\Omega)}^{2}+\left\|Y \mathbb{1}_{\llbracket 0, T_{n} \rrbracket}\right\|_{L^{p}\left(\Omega ; L^{2}(0, T ; V)\right)}^{2}+\left\|\xi X \mathbb{1}_{\llbracket 0, T_{n} \rrbracket}\right\|_{L^{p / 2}\left(\Omega ; L^{1}((0, T) \times D)\right)} \lesssim 1+\left\|X_{0}\right\|_{L^{p}(\Omega ; H)}^{2} .
$$


As the implicit constant is independent of $n$ and $T_{n}$ increases to $T$, we get

$$
\|Y\|_{L^{p}(\Omega ; C([0, T] ; H))}^{2}+\|Y\|_{L^{p}\left(\Omega ; L^{2}(0, T ; V)\right)}^{2}+\|\xi X\|_{L^{p / 2}\left(\Omega ; L^{1}((0, T) \times D)\right)} \lesssim 1+\left\|X_{0}\right\|_{L^{p}(\Omega ; H)}^{2} .
$$

The proof is completed noting that, for $E:=C([0, T] ; H) \cap L^{2}(0, T ; V)$,

$$
\|X\|_{E} \leq e^{\alpha T}\|Y\|_{E}
$$

If $B$ is Lipschitz-continuous, related arguments show that the solution map is Lipschitz-continuous between spaces with finite $p$-th moment in the whole range $p \in[0, \infty[$. We consider the cases $p>0$ and $p=0$ separately.

Proposition 5.2. Let $p>0$. If $B$ is Lipschitz-continuous in the sense of assumption (B1), then the solution map

$$
\begin{aligned}
& L^{p}(\Omega ; H) \longrightarrow L^{p}(\Omega ; C([0, T] ; H)) \cap L^{p}\left(\Omega ; L^{2}(0, T ; V)\right) \\
& X_{0} \longmapsto X
\end{aligned}
$$

is Lipschitz-continuous.

Proof. Let $X_{0}, Y_{0} \in L^{p}(\Omega ; H)$. The previous theorem asserts that the (unique) strong solutions $(X, \xi)$ and $(Y, \eta)$ to (1.1) with initial condition $X_{0}$ and $Y_{0}$, respectively, belong to $L^{p}(\Omega ; E)$, where, as before, $E$ stands for $C([0, T] ; H) \cap L^{2}(0, T ; V)$. By Itô's formula,

$$
\begin{aligned}
\|X-Y\|^{2} & +2 \int_{0}^{t}\langle A(X-Y), X-Y\rangle d s+2 \int_{0}^{t} \int_{D}(\xi-\eta)(X-Y) d s \\
= & \left\|X_{0}-Y_{0}\right\|^{2}+\int_{0}^{t}\|B(X)-B(Y)\|_{\mathscr{L}^{2}(U, H)}^{2} d s \\
& +2 \int_{0}^{t}(X-Y)(B(X)-B(Y)) d W
\end{aligned}
$$

where the third term on the left-hand side is positive by monotonicity of $\beta$. Let $\alpha>0$ be a constant to be chosen later, and set $X_{\alpha}:=X e^{-\alpha \cdot}, Y_{\alpha}:=Y e^{-\alpha}$. It follows by the integration-by-parts formula, in complete analogy to the proof of the previous theorem, by the Lipschitz continuity of $B$, and by the coercivity of $A$, that

$$
\begin{gathered}
\left\|X_{\alpha}-Y_{\alpha}\right\|^{2}+\alpha \int_{0}^{t}\left\|X_{\alpha}-Y_{\alpha}\right\|^{2} d s+\int_{0}^{t}\left\|X_{\alpha}-Y_{\alpha}\right\|_{V}^{2} d s \\
\lesssim\left\|X_{0}-Y_{0}\right\|^{2}+\int_{0}^{t}\left\|X_{\alpha}-Y_{\alpha}\right\|^{2} d s+M
\end{gathered}
$$

where $M:=\left(e^{-2 \alpha \cdot}(X-Y)(B(X)-B(Y))\right) \cdot W$. Taking supremum in time and the $L^{p / 2}(\Omega)$-(quasi)norm yields

$$
\begin{gathered}
\left\|X_{\alpha}-Y_{\alpha}\right\|_{L^{p}(\Omega ; C([0, T] ; H))}^{2}+\alpha\left\|X_{\alpha}-Y_{\alpha}\right\|_{L^{p}\left(\Omega ; L^{2}(0, T ; H)\right)}^{2}+\left\|X_{\alpha}-Y_{\alpha}\right\|_{L^{p}\left(\Omega ; L^{2}(0, T ; V)\right)}^{2} \\
\lesssim\left\|X_{0}-Y_{0}\right\|_{L^{p}(\Omega ; H)}^{2}+\left\|X_{\alpha}-Y_{\alpha}\right\|_{L^{p}\left(\Omega ; L^{2}(0, T ; H)\right)}^{2}+\left\|M_{T}^{*}\right\|_{L^{p / 2}(\Omega)},
\end{gathered}
$$

where, by Lemma 4.1

$$
\left\|M_{T}^{*}\right\|_{L^{p / 2}(\Omega)} \lesssim \varepsilon\left\|X_{\alpha}-Y_{\alpha}\right\|_{L^{p}(\Omega ; C([0, T] ; H))}^{2}+N(\varepsilon)\left\|X_{\alpha}-Y_{\alpha}\right\|_{L^{p}\left(\Omega ; L^{2}(0, T ; H)\right)}^{2}
$$

for any $\varepsilon>0$. Choosing first $\varepsilon$ small enough, then $\alpha$ sufficiently large, we obtain

$$
\left\|X_{\alpha}-Y_{\alpha}\right\|_{L^{p}(\Omega ; C([0, T] ; H))}^{2}+\left\|X_{\alpha}-Y_{\alpha}\right\|_{L^{p}\left(\Omega ; L^{2}(0, T ; V)\right)}^{2} \lesssim\left\|X_{0}-Y_{0}\right\|_{L^{p}(\Omega ; H)}^{2},
$$

which completes the proof noting that $\|X-Y\|_{E} \leq e^{\alpha T}\left\|X_{\alpha}-Y_{\alpha}\right\|_{E}$. 
Lipschitz continuity of the solution map can also be obtained in the case $p=0$. As already seen, the space $E:=C([0, T] ; H) \cap L^{2}(0, T ; V)$, equipped with the norm

$$
\|u\|_{E}:=\|u\|_{C([0, T] ; H)}+\|u\|_{L^{2}(0, T ; V)},
$$

is a Banach space. Then $L^{0}(\Omega ; E)$, endowed with the topology of convergence in probability, is a complete metrizable topological vector space. In particular, the distance

$$
d(f, g):=\mathbb{E}\left(\|f-g\|_{E} \wedge 1\right)
$$

generates its topology.

Proposition 5.3. If B is Lipschitz-continuous in the sense of assumption (B1), then the solution map

$$
\begin{aligned}
L^{0}(\Omega ; H) & \longrightarrow L^{0}(\Omega ; E) \\
X_{0} & \longmapsto X
\end{aligned}
$$

is Lipschitz-continuous.

Proof. Let $X_{0}, Y_{0} \in L^{0}\left(\Omega, \mathscr{F}_{0}, \mathbb{P} ; H\right)$, and $(X, \xi),(Y, \eta)$ the unique solutions in $\mathscr{J}_{0}$ to equation (1.1) with initial datum $X_{0}$ and $Y_{0}$, respectively. The stopping time

$$
T_{1}:=\inf \left\{t \geq 0:(X-Y)_{t}^{*}+\left(\int_{0}^{t}\|X(s)-Y(s)\|_{V}^{2} d s\right)^{1 / 2} \geq 1\right\} \wedge T .
$$

is well defined thanks to the pathwise continuity of $X$ and $Y$. For every $\alpha>0$, using the same notation as in the previous proof, Theorem 3.2 yields, by monotonicity of $\beta$ and coercivity of $A$,

$$
\begin{gathered}
\left(X_{\alpha}-Y_{\alpha}\right)_{t}^{* 2}+\int_{0}^{t}\left\|X_{\alpha}(s)-Y_{\alpha}(s)\right\|_{V}^{2} d s+\alpha \int_{0}^{t}\left\|X_{\alpha}(s)-Y_{\alpha}(s)\right\|^{2} d s \\
\lesssim\left\|X_{0}-Y_{0}\right\|^{2}+\int_{0}^{t}\left\|(B(X(s))-B(Y(s)))_{\alpha}\right\|_{\mathscr{L}^{2}(U, H)}^{2} d s \\
+\left(\left(X_{\alpha}-Y_{\alpha}\right)(B(X)-B(Y))_{\alpha} \cdot W\right)_{t}^{*}
\end{gathered}
$$

Raising to the power $1 / 2$, stopping at $T_{1}$, and taking expectation, we get, by the Lipschitz continuity of $B$,

$$
\begin{aligned}
& \mathbb{E}\left(X_{\alpha}-Y_{\alpha}\right)_{T_{1}}^{*}+\mathbb{E}\left(\int_{0}^{T_{1}}\left\|X_{\alpha}(s)-Y_{\alpha}(s)\right\|_{V}^{2} d s\right)^{1 / 2}+\sqrt{\alpha} \mathbb{E}\left(\int_{0}^{T_{1}}\left\|X_{\alpha}(s)-Y_{\alpha}(s)\right\|^{2} d s\right)^{1 / 2} \\
& \lesssim \mathbb{E} \mathbb{1}_{\llbracket 0, T_{1} \rrbracket}\left\|X_{0}-Y_{0}\right\|+\mathbb{E}\left(\int_{0}^{T_{1}}\left\|X_{\alpha}(s)-Y_{\alpha}(s)\right\|^{2} d s\right)^{1 / 2} \\
&+ \mathbb{E}\left(\left(X_{\alpha}-Y_{\alpha}\right)(B(X)-B(Y))_{\alpha} \cdot W\right)_{T_{1}}^{* 1 / 2},
\end{aligned}
$$

where, by Lemma 4.1 and Lipschitz continuity of $B$, the last term on the right-hand side is bounded by

$$
\varepsilon \mathbb{E}\left(X_{\alpha}-Y_{\alpha}\right)_{T_{1}}^{*}+N(\varepsilon) \mathbb{E}\left(\int_{0}^{T_{1}}\left\|X_{\alpha}(s)-Y_{\alpha}(s)\right\|^{2} d s\right)^{1 / 2}
$$

for every $\varepsilon>0$. Therefore, choosing $\varepsilon$ small enough and $\alpha$ large enough, we are left with

$$
\mathbb{E}(X-Y)_{T_{1}}^{*}+\mathbb{E}\left(\int_{0}^{T_{1}}\|X(s)-Y(s)\|_{V}^{2} d s\right)^{1 / 2} \lesssim \mathbb{E} \mathbb{1}_{\llbracket 0, T_{1} \rrbracket}\left\|X_{0}-Y_{0}\right\| .
$$

The proof is concluded noting that, by definition of $T_{1}$,

$$
(X-Y)_{T_{1}}^{*}+\left(\int_{0}^{T_{1}}\|X(s)-Y(s)\|_{V}^{2} d s\right)^{1 / 2}=\|X-Y\|_{C([0, T] ; H) \cap L^{2}(0, T ; V)} \wedge 1,
$$

and $\left\|X_{0}-Y_{0}\right\| \leq 1$ on $\llbracket 0, T_{1} \rrbracket$, hence

$$
\mathbb{E} \mathbb{1}_{\llbracket 0, T_{1} \rrbracket}\left\|X_{0}-Y_{0}\right\|=\mathbb{E} \mathbb{1}_{\llbracket 0, T_{1} \rrbracket}\left(\left\|X_{0}-Y_{0}\right\| \wedge 1\right) \leq \mathbb{E}\left(\left\|X_{0}-Y_{0}\right\| \wedge 1\right) .
$$




\section{A regularity result}

We are going to show that the regularity of the solution to equation (1.1) improves, if the initial datum and the diffusion coefficient are smoother, irrespective of the (possible) singularity of the drift coefficient $\beta$. In particular, we provide sufficient conditions implying that the variational solution to (1.1) is also an analytically strong solution, in the sense that it takes values in the domain of the part of $A$ in $H$ (see 83). If the solution to (1.1) generates a Markovian semigroup on $C_{b}(H)$ admitting an invariant measure, we also show that improved regularity of the solution carries over to further regularity of the invariant measure, in the sense that its support is made of smoother functions.

Theorem 6.1. Assume that the hypotheses of 2.2 are satisfied, that $A$ is symmetric and that

$$
X_{0} \in L^{2}\left(\Omega, \mathscr{F}_{0}, \mathbb{P} ; V\right), \quad B(\cdot, X) \in L^{2}\left(\Omega ; L^{2}\left(0, T ; \mathscr{L}^{2}(U, V)\right)\right) .
$$

Then the unique solution $(X, \xi)$ to the equation (1.1) satisfies

$$
X \in L^{2}(\Omega ; C([0, T] ; H)) \cap L^{2}\left(\Omega ; L^{\infty}(0, T ; V)\right) \cap L^{2}\left(\Omega ; L^{2}\left(0, T ; \mathrm{D}\left(A_{2}\right)\right)\right) .
$$

For the proof we need the following positivity result.

Lemma 6.2. Let $A_{\lambda}$ and $\beta_{\lambda}$ be the Yosida approximations of $A_{2}$ and $\beta$, respectively. One has

$$
\left\langle A_{\lambda} u, \beta_{\lambda}(u)\right\rangle \geq 0 \quad \forall u \in H .
$$

Proof. Let $j_{\lambda}: \mathbb{R} \rightarrow \mathbb{R}$ be the positive convex function defined as $j_{\lambda}(x):=\int_{0}^{x} \beta_{\lambda}(y) d y$. Then, for any $u, v \in L^{2}(D)$,

$$
j_{\lambda}(v)-j_{\lambda}(u) \geq j_{\lambda}^{\prime}(u)(v-u)
$$

a.e. in $D$, hence, integrating over $D$,

$$
\int_{D} j_{\lambda}(v)-\int j_{\lambda}(u) \geq\left\langle\beta_{\lambda}(u), v-u\right\rangle .
$$

Choosing $v=\left(I+\lambda A_{2}\right)^{-1} u$, one has $u-v=\lambda A_{\lambda} u$, thus also

$$
\lambda\left\langle\beta_{\lambda}(u), A_{\lambda} u\right\rangle \geq \int j_{\lambda}(u)-\int j_{\lambda}\left(\left(I+\lambda A_{2}\right)^{-1} u\right) .
$$

Since $A_{1}$ is an extension of $A_{2}$ and $u \in L^{1}(D)$, Jensen's inequality for sub-Markovian operators and accretivity of $A_{1}$ in $L^{1}(D)$ imply

$$
\int j_{\lambda}\left(\left(I+\lambda A_{2}\right)^{-1} u\right) \leq \int\left(I+\lambda A_{2}\right)^{-1} j_{\lambda}(u) \leq \int j_{\lambda}(u) .
$$

Proof of Theorem 6.1. For any $\lambda>0$, let $J_{\lambda}$ and $A_{\lambda}$ be the resolvent and the Yosida approximations of $A_{2}$, the part of $A$ in $H$, as defined in $\$ 3$ That is,

$$
J_{\lambda}:=\left(I+\lambda A_{2}\right)^{-1}, \quad A_{\lambda}:=\frac{1}{\lambda}\left(I-J_{\lambda}\right) .
$$

We recall that $J_{\lambda}$ and $A_{\lambda}$ are bounded linear operators on $H$, that $J_{\lambda}$ is a contraction, and that $A_{\lambda}=A J_{\lambda}$.

Setting $G:=B(\cdot, X)$, let us consider the equation

$$
d X_{\lambda}(t)+A_{\lambda} X_{\lambda}(t) d t+\beta_{\lambda}\left(X_{\lambda}(t)\right) d t=G(t) d W(t), \quad X_{\lambda}(0)=X_{0} .
$$

Since $A_{\lambda}$ is bounded and $\beta_{\lambda}$ is Lipschitz-continuous, it admits a unique strong solution

$$
X_{\lambda} \in L^{2}(\Omega ; C([0, T] ; H)),
$$

for which Itô's formula for the square of the $H$-norm yields

$$
\begin{array}{r}
\frac{1}{2}\left\|X_{\lambda}(t)\right\|^{2}+\int_{0}^{t}\left\langle A_{\lambda} X_{\lambda}(s), X_{\lambda}(s)\right\rangle d s+\int_{0}^{t} \int_{D} \beta_{\lambda}\left(X_{\lambda}(s)\right) X_{\lambda}(s) d s \\
=\frac{1}{2}\left\|X_{0}\right\|^{2}+\frac{1}{2} \int_{0}^{t}\|G(s)\|_{\mathscr{L}^{2}(U, H)}^{2} d s+\int_{0}^{t} X_{\lambda}(s) G(s) d W(s)
\end{array}
$$


for all $t \in[0, T] \mathbb{P}$-almost surely. Writing

$$
X_{\lambda}=J_{\lambda} X_{\lambda}+X_{\lambda}-J_{\lambda} X_{\lambda}=J_{\lambda} X_{\lambda}+\lambda A_{\lambda} X_{\lambda}
$$

and recalling that $A_{\lambda}=A J_{\lambda}$ and that $A$ is coercive, we have, after taking supremum in time and expectation,

$$
\begin{aligned}
\frac{1}{2} \mathbb{E}\left\|X_{\lambda}\right\|_{C([0, T] ; H)}^{2}+C \mathbb{E} \int_{0}^{T}\left\|J_{\lambda} X_{\lambda}(s)\right\|_{V}^{2} d s & \\
& +\lambda \mathbb{E} \int_{0}^{T}\left\|A_{\lambda} X_{\lambda}(s)\right\|^{2} d s+\mathbb{E} \int_{0}^{T} \int_{D} \beta_{\lambda}\left(X_{\lambda}(s)\right) X_{\lambda}(s) d s \\
\leq & \frac{1}{2} \mathbb{E}\left\|X_{0}\right\|^{2}+\frac{1}{2} \mathbb{E} \int_{0}^{T}\|G(s)\|_{\mathscr{L}^{2}(U, H)}^{2} d s+\mathbb{E} \sup _{t \in[0, T]}\left|\int_{0}^{t} X_{\lambda}(s) G(s) d W(s)\right|,
\end{aligned}
$$

where, by Lemma 4.1, the last term on the right-hand side is bounded by

$$
\varepsilon \mathbb{E}\left\|X_{\lambda}\right\|_{C([0, T] ; H)}^{2}+C_{\varepsilon} \mathbb{E} \int_{0}^{T}\|G(s)\|_{\mathscr{L}^{2}(U, H)}^{2} d s,
$$

so that, rearranging terms and choosing $\varepsilon$ small enough, we deduce that there exists a constant $N>0$ independent of $\lambda$ such that

$$
\left\|X_{\lambda}\right\|_{L^{2}(\Omega ; C([0, T] ; H))}^{2}+\left\|J_{\lambda} X_{\lambda}\right\|_{L^{2}\left(\Omega ; L^{2}(0, T ; V)\right)}^{2}+\left\|\beta_{\lambda}\left(X_{\lambda}\right) X_{\lambda}\right\|_{L^{1}(\Omega \times(0, T) \times D)}<N .
$$

Moreover, let us introduce the function

$$
\begin{aligned}
\varphi_{\lambda}: H & \longrightarrow[0,+\infty[, \\
u & \longmapsto \frac{1}{2}\left\langle A_{\lambda} u, u\right\rangle .
\end{aligned}
$$

The linearity, boundedness and symmetry of $A_{\lambda}$ immediately imply that $\varphi_{\lambda} \in C^{2}(H)$ with $D \varphi_{\lambda}(u)=$ $A_{\lambda}$, and, by linearity, $D^{2} \varphi_{\lambda}(u)=A_{\lambda}$, for all $u \in H$ (in the latter statement $A_{\lambda}$ has to be considered as an element of $\mathscr{L}_{2}(H)$, the space of bounded bilinear maps on $\left.H\right)$. Itô's formula applied to $\varphi_{\lambda}\left(X_{\lambda}\right)$ then yields

$$
\begin{aligned}
\varphi_{\lambda}\left(X_{\lambda}(t)\right) & +\int_{0}^{t}\left\|A_{\lambda} X_{\lambda}(s)\right\|^{2} d s+\int_{0}^{t}\left\langle A_{\lambda} X_{\lambda}(s), \beta_{\lambda}\left(X_{\lambda}(s)\right)\right\rangle d s \\
= & \varphi_{\lambda}\left(X_{0}\right)+\frac{1}{2} \int_{0}^{t} \operatorname{Tr}\left(G^{*}(s) D^{2} \varphi_{\lambda}\left(X_{\lambda}(s)\right) G(s)\right) d s+\int_{0}^{t} A_{\lambda} X_{\lambda}(s) G(s) d W(s)
\end{aligned}
$$

for every $t \in[0, T] \mathbb{P}$-almost surely. Writing, as before, $X_{\lambda}=J_{\lambda} X_{\lambda}+\lambda A_{\lambda} X_{\lambda}$, the coercivity of $A$ implies that

$$
\varphi_{\lambda}\left(X_{\lambda}\right)=\frac{1}{2}\left\langle A_{\lambda} X_{\lambda}, X_{\lambda}\right\rangle \geq \frac{C}{2}\left\|J_{\lambda} X_{\lambda}\right\|_{V}^{2}+\frac{1}{2} \lambda\left\|A_{\lambda} X_{\lambda}\right\|^{2} \gtrsim\left\|J_{\lambda} X_{\lambda}\right\|_{V}^{2} .
$$

The continuity of $J_{\lambda}$ on $V$ (see Lemma 3.1) instead implies

$$
\varphi_{\lambda}\left(X_{0}\right)=\left\langle A J_{\lambda} X_{0}, X_{0}\right\rangle \leq\|A\|_{\mathscr{L}\left(V, V^{\prime}\right)}\left\|J_{\lambda} X_{0}\right\|_{V}\left\|X_{0}\right\|_{V} \lesssim\|A\|_{\mathscr{L}\left(V, V^{\prime}\right)}\left\|X_{0}\right\|_{V}^{2}
$$

Denoting a complete orthonormal basis of $U$ by $\left(u_{k}\right)_{k}$, we have, recalling again the continuity of $J_{\lambda}$ on $V$ and that $D^{2} \varphi_{\lambda}(u)=A_{\lambda}$ for all $u \in H$,

$$
\begin{aligned}
\operatorname{Tr}\left(G^{*} D^{2} \varphi_{\lambda}\left(X_{\lambda}\right) G\right) & =\sum_{k=0}^{\infty}\left\langle G^{*} D^{2} \varphi_{\lambda}\left(X_{\lambda}\right) G u_{k}, u_{k}\right\rangle_{U}=\sum_{k=0}^{\infty}\left\langle A_{\lambda} G u_{k}, G u_{k}\right\rangle \\
& =\sum_{k=0}^{\infty}\left\langle A J_{\lambda} G u_{k}, G u_{k}\right\rangle \leq\|A\|_{\mathscr{L}\left(V, V^{\prime}\right)} \sum_{k=0}^{\infty}\left\|J_{\lambda} G u_{k}\right\|_{V}\left\|G u_{k}\right\|_{V} \\
& \lesssim\|A\|_{\mathscr{L}\left(V, V^{\prime}\right)} \sum_{k=0}^{\infty}\left\|G u_{k}\right\|_{V}^{2}=\|A\|_{\mathscr{L}\left(V, V^{\prime}\right)}\|G\|_{\mathscr{L}^{2}(U, V)}^{2} .
\end{aligned}
$$


Moreover, by Lemma 4.1

$$
\begin{aligned}
\mathbb{E}\left(\left(A_{\lambda} X_{\lambda} G\right) \cdot W\right)_{T}^{*} & \lesssim \varepsilon \mathbb{E} \sup _{t \in[0, T]}\left\|A_{\lambda} X_{\lambda}(t)\right\|_{V^{\prime}}^{2}+N(\varepsilon) \mathbb{E} \int_{0}^{T}\|G(s)\|_{\mathscr{L}^{2}(U, V)}^{2} d s \\
& \leq \varepsilon\|A\|_{\mathscr{L}\left(V, V^{\prime}\right)}^{2} \mathbb{E} \sup _{t \in[0, T]}\left\|J_{\lambda} X_{\lambda}(t)\right\|_{V}^{2}+N(\varepsilon) \mathbb{E} \int_{0}^{T}\|G(s)\|_{\mathscr{L}^{2}(U, V)}^{2} d s
\end{aligned}
$$

for every $\varepsilon>0$. Taking supremum in time and expectations in the Itô formula for $\varphi_{\lambda}\left(X_{\lambda}\right)$, choosing $\varepsilon$ small enough we obtain, thanks to the previous lemma and hypothesis (6.1), that there exists a constant $N>0$ independent of $\lambda$, such that

$$
\left\|J_{\lambda} X_{\lambda}\right\|_{L^{2}\left(\Omega ; L^{\infty}(0, T ; V)\right)}^{2}+\left\|A_{\lambda} X_{\lambda}\right\|_{L^{2}\left(\Omega ; L^{2}(0, T ; H)\right)}^{2}<N .
$$

Reasoning as in 24], it follows by (6.2) that

$$
\begin{aligned}
X_{\lambda} \longrightarrow X & \text { weakly in } L^{2}\left(\Omega ; L^{2}(0, T ; H)\right), \\
J_{\lambda} X_{\lambda} \longrightarrow X & \text { weakly in } L^{2}\left(\Omega ; L^{2}(0, T ; V)\right), \\
\beta_{\lambda}\left(X_{\lambda}\right) \longrightarrow \xi & \text { weakly in } L^{1}(\Omega \times(0, T) \times D),
\end{aligned}
$$

where $(X, \xi)$ is the unique solution to (1.1). Moreover, by (6.3) we have

$$
J_{\lambda} X_{\lambda}-X_{\lambda}=\lambda A_{\lambda} X_{\lambda} \longrightarrow 0 \quad \text { in } L^{2}\left(\Omega ; L^{2}(0, T ; H)\right),
$$

hence, $\mathbb{P}$-almost surely and for almost every $t \in(0, T), J_{\lambda} X_{\lambda}(t)$ converges to $X(t)$ in $H$. Since the function $\|\cdot\|_{V}^{2}$ is lower semicontinuous on $H$, we infer that

$$
\|X(t)\|_{V}^{2} \leq \liminf _{\lambda \rightarrow 0}\left\|J_{\lambda} X_{\lambda}(t)\right\|_{V}^{2}
$$

for almost every $t$. Hence, taking supremum in time and expectation, we deduce that

$$
X \in L^{2}\left(\Omega ; L^{\infty}(0, T ; V)\right) .
$$

Moreover, by (6.3) we also have

$$
A_{\lambda} X_{\lambda} \longrightarrow \eta \quad \text { weakly in } L^{2}\left(\Omega ; L^{2}(0, T ; H)\right),
$$

hence, since $J_{\lambda} X_{\lambda} \rightarrow X$ weakly in $L^{2}\left(\Omega ; L^{2}(0, T ; V)\right)$, by the continuity and the linearity of $A$ we necessarily have $\eta=A X$. In particular, $X \in L^{2}\left(\Omega ; L^{2}\left(0, T ; \mathrm{D}\left(A_{2}\right)\right)\right)$.

As last result we show that if the solution to (1.1) generates a Markovian semigroup $P=\left(P_{t}\right)_{t \geq 0}$ on $C_{b}(H)$ admitting an invariant measure, then the improved regularity of solutions given by Theorem 6.1 implies better integrability properties also for the invariant measures. Existence and uniqueness of invariant measures, ergodicity, and the Kolmogorov equation associated to (1.1) were studied in 20. In particular, we recall the following result (see [20, §5]). The set of invariant measures of $P$ will be denoted by $\mathscr{M}$.

Proposition 6.3. Assume that the the hypotheses of 2.2 are satisfied, that $X_{0} \in H$ is non-random, and that $B$ is non-random and time-independent. Then the solution $X$ to (1.1) is Markovian and its associated transition semigroup $P$ admits an ergodic invariant measure. Moreover, there exists a positive constant $N$ such that

$$
\int_{H}\|u\|_{V}^{2} \mu(d u)+\int_{H} \int_{D} j(u) \mu(d u)+\int_{H} \int_{D} j^{*}\left(\beta^{0}(u)\right) \mu(d u)<N \quad \forall \mu \in \mathscr{M} .
$$

If $\beta$ is superlinear, there exists a unique invariant measure for $P$, which is strongly mixing as well. 
Theorem 6.4. Assume that the hypotheses of 2.2 are satisfied, that $A$ is symmetric and that

$$
B: H \rightarrow \mathscr{L}^{2}(U, V), \quad\|B(v)\|_{\mathscr{L}^{2}(U, V)} \lesssim 1+\|v\|_{V} .
$$

Then there exists a positive constant $N$ such that

$$
\int_{H}\|A u\|^{2} \mu(d u)<N \quad \forall \mu \in \mathscr{M}
$$

In particular, every invariant measure $\mu$ is concentrated on $\mathrm{D}\left(A_{2}\right)$, that is $\mu\left(\mathrm{D}\left(A_{2}\right)\right)=1$.

Proof. For every $x \in V$, let $\left(X^{x}, \xi^{x}\right)$ be the unique strong solution to (1.1) with initial datum $x$. Setting $G:=B\left(X^{x}\right)$, Itô's formula for $\varphi_{\lambda}\left(X_{\lambda}\right)$ as in the proof of the previous theorem yields

$$
\begin{aligned}
& \varphi_{\lambda}\left(X_{\lambda}(t)\right)+\int_{0}^{t}\left\|A_{\lambda} X_{\lambda}(s)\right\|^{2} d s+\int_{0}^{t}\left\langle A_{\lambda} X_{\lambda}(s), \beta_{\lambda}\left(X_{\lambda}(s)\right)\right\rangle d s \\
& =\varphi_{\lambda}(x)+\frac{1}{2} \int_{0}^{t} \operatorname{Tr}\left(G^{*}(s) D^{2} \varphi_{\lambda}\left(X_{\lambda}(s)\right) G(s)\right) d s+\int_{0}^{t} A_{\lambda} X_{\lambda}(s) G(s) d W(s) .
\end{aligned}
$$

Since $A_{\lambda} X_{\lambda} \in L^{2}\left(\Omega ; L^{\infty}(0, T ; H)\right)$ and $G \in L^{2}\left(\Omega ; L^{2}\left(0, T ; \mathscr{L}^{2}(U, H)\right)\right)$, the last term on the right hand side is a martingale; hence, taking expectations and recalling that $\varphi_{\lambda}(x) \lesssim\|x\|_{V}^{2}$, it follows by Lemma 6.2 and by the estimates obtained in the proof of the previous theorem that

$$
\mathbb{E} \int_{0}^{t}\left\|A_{\lambda} X_{\lambda}(s)\right\|^{2} d s \lesssim\|x\|_{V}^{2}+\mathbb{E}\|G\|_{L^{2}\left(0, t ; \mathscr{L}^{2}(U, V)\right)}^{2}
$$

Since this holds for every $\lambda>0$, letting $\lambda \rightarrow 0$ and recalling that, as in the proof of the previous theorem, $A_{\lambda} X_{\lambda}$ converges to $A X$ weakly in $L^{2}\left(\Omega ; L^{2}(0, T ; H)\right)$, a weak lower semicontinuity argument and the linear growth assumption on $B$ yield

$$
\mathbb{E} \int_{0}^{t}\left\|A X^{x}(s)\right\|^{2} d s \lesssim 1+\|x\|_{V}^{2}
$$

for every $t \in[0, T]$ and $x \in V$. Let us introduce the function $F: H \rightarrow[0,+\infty]$ defined as

$$
F(u):= \begin{cases}\|A u\|^{2} & \text { if } u \in \mathrm{D}\left(A_{2}\right), \\ +\infty & \text { if } u \in H \backslash \mathrm{D}\left(A_{2}\right),\end{cases}
$$

and the sequence of functions $\left(F_{n}\right)_{n \in \mathbb{N}}, F_{n}: H \rightarrow[0,+\infty)$, defined as

$$
F_{n}(u):=\left\|A_{1 / n} u\right\|^{2} \wedge n^{2} .
$$

It is easily seen that $F_{n} \in C_{b}(H)$ for all $n \in \mathbb{N}$ and that $F_{n}$ converges pointwise to $F$ from below. Therefore, for any invariant measure $\mu$, it follows by Fubini's theorem that

$$
\begin{aligned}
\int_{H} F_{n}(x) \mu(d x) & =\int_{0}^{1} \int_{H} F_{n}(x) \mu(d x) d s=\int_{0}^{1} \int_{H} P_{s} F_{n}(x) \mu(d x) d s \\
& =\int_{H} \int_{0}^{1} \mathbb{E}\left(\left\|A_{1 / n} X^{x}(s)\right\|^{2} \wedge n^{2}\right) d s \mu(d x) \\
& \leq \int_{H} \mathbb{E} \int_{0}^{1}\left\|A_{1 / n} X^{x}(s)\right\|^{2} d s \mu(d x)
\end{aligned}
$$

Recalling that $\left\|A_{\lambda} u\right\| \leq\|A u\|$ for all $u \in H$, it follows by 6.4 that

$$
\int_{H} F_{n}(x) \mu(d x) \lesssim 1+\int_{H}\|x\|_{V}^{2} \mu(d x)
$$

Since $\|\cdot\|_{V}^{2} \in L^{1}(H, \mu)$ by [20. Theorem 5.3], we get

$$
\int_{H} F_{n}(x) \mu(d x) \lesssim N
$$

for a positive constant $N$, independent of $n$ and $\mu$. Letting $n \rightarrow \infty$, by the monotone convergence theorem we deduce that $F \in L^{1}(H, \mu)$, hence $F$ is finite $\mu$-almost everywhere in $H$, and in particular $\mu\left(\mathrm{D}\left(A_{2}\right)\right)=1$. 


\section{References}

[1] R. Anton, D. Cohen, and L. Quer-Sardanyons, A fully discrete approximation of the onedimensional stochastic heat equation, arXiv:1711.08340.

[2] W. Arendt, R. Chill, C. Seifert, D. Vogt, and J. Voigt, Form methods for evolution equations and applications, Lecture Notes of the 18th Internet Seminar on Evolution Equations, 2015.

[3] V. Barbu, Existence for semilinear parabolic stochastic equations, Atti Accad. Naz. Lincei Cl. Sci. Fis. Mat. Natur. Rend. Lincei (9) Mat. Appl. 21 (2010), no. 4, 397-403. MR 2746091 (2012d:35424)

[4] - Nonlinear differential equations of monotone types [sic] in Banach spaces, Springer, New York, 2010. MR 2582280

[5] V. Barbu, G. Da Prato, and M. Röckner, Existence of strong solutions for stochastic porous media equation under general monotonicity conditions, Ann. Probab. 37 (2009), no. 2, 428-452. MR MR2510012

[6] A. Bensoussan and A. Rascanu, Stochastic variational inequalities in infinite-dimensional spaces, Numer. Funct. Anal. Optim. 18 (1997), no. 1-2, 19-54. MR 1442017

[7] H. Brézis, Functional analysis, Sobolev spaces and partial differential equations, Springer, New York, 2011. MR 2759829 (2012a:35002)

[8] M. Emery, Équations différentielles stochastiques lipschitziennes: étude de la stabilité, Séminaire de Probabilités, XIII (Univ. Strasbourg, Strasbourg, 1977/78), Lecture Notes in Math., vol. 721, Springer, Berlin, 1979, pp. 281-293. MR 544801 (81h:60075)

[9] H. Garcke and Kei Fong Lam, Well-posedness of a Cahn-Hilliard system modelling tumour growth with chemotaxis and active transport, European J. Appl. Math. 28 (2017), no. 2, 284-316. MR 3613312

[10] H. Garcke, Kei Fong Lam, and E. Rocca, Optimal control of treatment time in a diffuse interface model of tumor growth, Appl. Math. Optim. 78 (2018), no. 3, 495-544. MR 3868741

[11] B. Gess, Strong solutions for stochastic partial differential equations of gradient type, J. Funct. Anal. 263 (2012), no. 8, 2355-2383. MR 2964686

[12] B. Gess and M. Röckner, Singular-degenerate multivalued stochastic fast diffusion equations, SIAM J. Math. Anal. 47 (2015), no. 5, 4058-4090. MR 3505171

[13] _ Stochastic variational inequalities and regularity for degenerate stochastic partial differential equations, Trans. Amer. Math. Soc. 369 (2017), no. 5, 3017-3045. MR 3605963

[14] I. Gyöngy, Lattice approximations for stochastic quasi-linear parabolic partial differential equations driven by space-time white noise. II, Potential Anal. 11 (1999), no. 1, 1-37. MR 1699161

[15] M. Haase, Convexity inequalities for positive operators, Positivity 11 (2007), no. 1, 57-68. MR 2297322 (2008d:39034)

[16] S. G. Kreĭn, Yu. İ. Petunīn, and E. M. Semënov, Interpolation of linear operators, Translations of Mathematical Monographs, vol. 54, American Mathematical Society, Providence, R.I., 1982. MR 649411 (84j:46103)

[17] N. V. Krylov, An analytic approach to SPDEs, Stochastic partial differential equations: six perspectives, Math. Surveys Monogr., vol. 64, Amer. Math. Soc., Providence, RI, 1999, pp. 185-242. MR MR1661766 (99j:60093)

[18] N. V. Krylov and B. L. Rozovskiŭ, Stochastic evolution equations, Current problems in mathematics, Vol. 14 (Russian), Akad. Nauk SSSR, Vsesoyuz. Inst. Nauchn. i Tekhn. Informatsii, Moscow, 1979, pp. 71-147, 256. MR MR570795 (81m:60116) 
[19] M. Kunze and J. van Neerven, Continuous dependence on the coefficients and global existence for stochastic reaction diffusion equations, J. Differential Equations 253 (2012), no. 3, 1036-1068. MR 2922662

[20] C. Marinelli and L. Scarpa, Ergodicity and Kolmogorov equations for dissipative SPDEs with singular drift: a variational approach, arXiv:1710.05612.

[21] _ Strong solutions to SPDEs with monotone drift in divergence form, arXiv:1612.08260.

[22] Well-posedness of monotone semilinear SPDEs with semimartingale noise, arXiv:1805.07562.

[23] _ A note on doubly nonlinear SPDEs with singular drift in divergence form, Atti Accad. Naz. Lincei Rend. Lincei Mat. Appl. 29 (2018), no. 4, 619-633. MR 3896258

[24] _ A variational approach to dissipative SPDEs with singular drift, Ann. Probab. 46 (2018), no. 3, 1455-1497. MR 3785593

[25] M. Métivier, Semimartingales, Walter de Gruyter \& Co., Berlin, 1982. MR MR688144 (84i:60002)

[26] E. Pardoux, Equations aux derivées partielles stochastiques nonlinéaires monotones, Ph.D. thesis, Université Paris XI, 1975.

[27] L. Scarpa, Well-posedness for a class of doubly nonlinear stochastic PDEs of divergence type, J. Differential Equations 263 (2017), no. 4, 2113-2156. MR 3650335

[28] - Optimal distributed control of a stochastic Cahn-Hilliard equation, SIAM J. Control Optim. 57 (2019), no. 5, 3571-3602. MR 4025767 